\title{
New data on tail lengths and variation along the caudal series in the non-avialan dinosaurs
}

\author{
David W E Hone ${ }^{\text {Corresp., } 1}$, W. Scott Persons ${ }^{2}$, Steven C Le Comber ${ }^{1}$ \\ ${ }^{1}$ School of Biological and Chemical Sciences, Queen Mary University of London, London, United Kingdom \\ 2 Mace Brown Museum of Natural History, College of Charleston, Charleston, South Carolina, United States \\ Corresponding Author: David W E Hone \\ Email address: dwe_hone@yahoo.com
}

The tails of non-avialan dinosaurs varied considerably in terms of overall length, total number of vertebrae, and gross form and function. A new dataset confirms that there is little or no consistent relationship between tail length and snout-sacrum length.

Consequently, attempts to estimate one from the other are likely to be very error-prone. Patterns of changes in centra lengths across the caudal series vary among non-avian dinosaurs. However, some overarching patterns do emerge. A number of taxa show (anterior to posterior) a series of short centra, followed by a series of longer centra, with the remainder of the tail consisting of a long series of centra tapering in length. This pattern is consistent with functional constraints, and the anterior series of longer centra are coincident with the major attachments of femoral musculature. This pattern is not present in many basal taxa and may have evolved independently in different dinosaurian groups, further suggesting functional importance. 


\section{New data on tail lengths and variation along the caudal series in}

\section{2 the non-avialan dinosaurs}

3

4 David W. E. Hone ${ }^{1}$, W. Scott Persons IV ${ }^{2}$, Steven Le Comber ${ }^{1}$

5

Corresponding Author:

David W. E Hone Road, London, E1 4NS, UK Email address: d.hone@qmul.ac.uk

\section{Abstract}

1. School of Biological and Chemical Sciences, Queen Mary University of London, Mile End Road, London, E1 4NS, UK.

2. Mace Brown Museum of Natural History, College of Charleston, Charleston, South Carolina 29424, USA

School of Biological and Chemical Sciences, Queen Mary University of London, Mile End

The tails of non-avialan dinosaurs varied considerably in terms of overall length, total number of vertebrae, and gross form and function. A new dataset confirms that there is little or no consistent relationship between tail length and snout-sacrum length. Consequently, attempts to estimate one from the other are likely to be very error-prone. Patterns of changes in centra lengths across the caudal series vary among non-avian dinosaurs. However, some overarching 
26 patterns do emerge. A number of taxa show (anterior to posterior) a series of short centra,

27 followed by a series of longer centra, with the remainder of the tail consisting of a long series of

28 centra tapering in length. This pattern is consistent with functional constraints, and the anterior

29 series of longer centra are coincident with the major attachments of femoral musculature. This

30 pattern is not present in many basal taxa and may have evolved independently in different

31 dinosaurian groups, further suggesting functional importance.

32

33

34

35

36

37

\section{Introduction}

The caudal vertebral series of the non-avialan dinosaurs (hereafter simply 'dinosaurs') were variable in form (Fig. 1) and served many roles. Dinosaur tails had a biomechanical function in locomotion (e.g. Hutchinson, Ng-Thow-Hing \& Anderson, 2007; Persons \& Currie, 2011a) and balance (e.g. Hutchinson \& Gatesy, 2001; Libby et al. 2012)), and some were specialized for behavioural roles including inter- and intraspecific combat (e.g. Mallison, 2011; Arbour 2009) and signaling (e.g. Persons, Currie \& Norell, 2014). Despite this importance, the osteological caudal anatomy of dinosaurs has received far less attention than most other major anatomical regions.

To date, few dinosaur tails have been identified that are truly complete (i.e. represented by every single vertebra in the series). Those that are complete show considerable variation both inter- and intraspecifically (Hone, 2012). This limits potential comparisons between taxa and the confidence of attempts to reconstruct tail forms for taxa that lack substantial caudal material, especially estimations of aspects like total length (Hone, 2012).

Studies of dinosaur tails have often focused on tails as flexible structures (Pittman et al., 2013) to support large muscle groups, in particular the caudofemoralis, which serves as a major 
51 driver in locomotion (Allen, Paxton \& Hutchinson, 2009; Persons \& Currie, 2011a). Previous

52 work has argued that the caudofemoralis has substantially influenced the form of anterior caudal

53 osteology, and adaptations suggested to be linked to the caudofemoralis include haemal spine

54 depth, prominent chevron and vertebral sulci (e.g., Persons and Currie 2011a, b; Cau and

55 Serventi 2017), and, most frequently, the 'transition point' of the lateral processes (Russell,

56 1972; Gauthier, 1986; Gatesy, 1990; Gatesy and Thomason, 1995; Persons and Currie 2011b).

57 The transition point is the region of the tail where the lateral processes end, and, by extension,

58 where the caudofemoralis is inferred to have terminated. The association of a functionally-

59 distinct major muscle set with one region of the tail suggests that dinosaur tails were modular,

60 with different tail regions functioning in different ways. Modularity is obviously true of

61 dinosaurs that bear highly-derived caudal features, such as pygostyles or tail-clubs. Such derived

62 and obvious specialisations notwithstanding, when compared to vertebrae from elsewhere in the

63 axial column, caudal vertebrae are generally simple in overall form. Dinosaur tail modularity

64 may be true more generally and be associated with less apparent morphological variations.

65 Based on these considerations, this paper focuses on variation in anteroposterior centrum

66 length. Little previous work has been done examining patterns of centrum lengths, although

67 centrum length has obvious influence on tail structure and, by extension, tail function. Recently,

68 Nuñez Demarco et al. (2018) demonstrated a general pattern of either decreasing lengths of

69 vertebrae along the tail, or an increase in length to the midpoint, followed by a decrease for a

70 number of extant reptiles. A reduction in the length of caudal vertebrae along the distal tail of the

71 extinct Mesosaurus was also demonstrated, though some individuals showed stability in lengths

72 distally (Nuñez Demarco et al., 2018). 
74 relative stiffness and stability to a tail (or at least parts of it), while a series of shorter vertebrae

75 would provide a zone of greater relative flexibility (e.g. see Persons, Currie \& Norrell, 2014).

76 Having short vertebrae means having more flexing points per unit of length. All other factors

77 remaining equal, a three-meter-long section of tail with thirty vertebrae is more flexible than a

78 three-meter-long section of tail with only twenty vertebrae.

It has been argued that the proportions of dorsal vertebrae are good correlates of absolute body size in tetrapod (see Currie 1978 for a review), but no similar argument has been made for caudal vertebrae. The total number of vertebrae is correlated with body size in many basal vertebrates (Head \& Polly, 2007), but less so in taxa where there is vertebral regionalization and functional constraint (as in birds and mammals - Wake, 1979). The tails however, may vary considerably, even in mammals (both in terms of caudal count and total length e.g., Garland, 1985; Cavallini, 1995; Alroy, 2019) suggesting it is relatively free of such constraints. In dinosaurs, changes in vertebral length down the caudal series have typically been

87 considered and described as simple sequential reductions (e.g. see Gilmore, 1936 on

88 Apatosaurus and Sereno, 1997 on Psittacosaurus). However, the proximal caudal vertebrae of Apatosaurus actually show sections of increase (in terms of both proportional and absolute centrum lengths - see below), and in the tail of Psittacosaurus, although the vertebrae never increase in absolute length, there are sections of length stability and decreases are not always 92 regular (Sereno, 1997).

94 Dinosauria, which make total body-length difficult to predict (following Hone, 2012). We would 95 also expect the tail structure of bipedal taxa differs from that of quadrupeds, as the tail is more 
96 important for balance in bipeds. We predict that centrum length does not follow a simple pattern

97 of decrease in length in successive vertebrae. Specifically, the presence of the lateral processes is

98 known to be associated with a major change in tail function (the presence of the caudofemoralis

99 musculature) and we, therefore, predict that the posterior loss of the lateral processes correlates

100 with a change in centrum lengths. It is expected that testing these hypotheses will reveal

101 variation that is important to the function and evolution of tails in different subclades and

102 locomotor regimes within Dinosauria.

103

104

105

106

\section{Institutional Abbreviations}

AMNH American Museum of Natural History, New York; BMNS Belgium Museum of Natural

107 Sciences, Brussels; CAGS Chinese Academy of Geological Sciences, Beijing; CM Carnegie

108 Museum of Natural History, Pittsburgh; CMN Canadian Museum of Nature, Aylmer; CYGYB /

109 CYNG Chaoyang Paleontological Museum, Chaoyang, Liaoning; FMNH Field Museum of

110 Natural History, Chicago; GIN / Gi-SPS Institute of Geology, Mongolian Academy of Sciences,

111 Ulan Baator; GMZ Grant Museum of Zoology, London; IGM Mongolian Academy of Sciences,

112 Ulan Baator; IVPP Institute of Vertebrate Paleontology and Paleoanthropology, Beijing; JME

113 Jura Museum, Eichstätt; JMP Henan Geological Museum, Henan Province; LPM Liaoning

114 Provincial Museum of Paleontology, Liaoning; MB.R. fossil reptiles collection of MfN (Museum

115 für Naturkunde) Berlin; MNA Museum of Northern Arizona, Flagstaff; MPC Mongolian

116 Paleontological Centre, Mongolian Academy of Sciences, Ulan Baator; NHMUK British

117 Museum of Natural History, London; OMNH Oklahoma Museum of Natural History, Norman;

118 PIN Paleontological Institute, Russian Academy of Sciences, Moscow; PMOL Paleontological

119 Museum of Liaoning, Shenyang Normal University, Shenyang; QM Qijiang Dinosaur National

120 Geological Park Museum, Liaoning; ROM Royal Ontario Museum, Toronto; RTMP Royal 
121 Tyrrell Museum of Palaeontology, Drumheller; SC Italian State Collections; SMA

122 Sauriermuseum Aathal, Aathal; UCMP University of California Museum of Paleontology,

123 Berkeley; USNM Smithsonian Museum of Natural History, Washington, DC; YPM Yale

124 Peabody Museum, New Haven; ZDM Zigong Dinosaur Museum, Zigong.

125

126

127

128

129

Materials \& Methods

We expanded on the dataset of Hone (2012), with additional measurement data collected directly from specimens, from photographs, and from the literature. Previously overlooked and new

130

material was identified by ourselves and also suggested by various sources (see

131 acknowledgements).

A complete tail was defined as one with every vertebra present down to the last caudal.

The last caudal can typically be identified by a rounded posterior face (this trait remains useful

even in procoelous vertebrae, because the extent of the rounding exceeds that observed

elsewhere in the series) and a lack of postzygopophyses and/or neural spine (Hone, 2012).

Additional tails were regarded as complete where, although one or more elements were not

preserved, the absent material could still be accurately recorded because there was either an impression of the missing material in the matrix or the missing material was bounded by other elements. Nearly complete tails were also included where it was felt that the missing material could be accurately reconstructed from other specimens of the same genus or species. For example, a total tail length was calculated and included where two or more individual specimens were complete enough to suggest the animals had very similar body sizes, and where the tails of

143 both included a series of overlapping elements (e.g. an anterior tail portion and a posterior tail

144 portion, with both possessing the last chevron or last lateral process pair). Total tail length was 
145 taken as the sum total of all individual caudal centra length measurements or as the single

146 measurement of the caudal series when centra were preserved closely appressed together. In

147 many cases, the live animals may have possessed intervertebral discs that would have increased

148 tail length, but these cannot be easily estimated and so were simply excluded (though see also

149 Rothschild et al., 2020 who argue for their absence).

150 Total femoral length was taken as a simple proxy for mass / body size (following Hone,

151 2012) for each specimen. Although other proxies (e.g. femur circumference - Campione \&

152 Evans, 2012) are stronger correlates of mass, femur length is appropriate for such datasets, and

153 length was the only consistently reliable measurement for samples from the literature and from

154 specimens that are taphonomically distorted. To examine the relationship between tail length and

155 body size, we compared snout to sacrum length and tail length using a simple linear least-squares

156 regression. Snout-sacrum length was taken as the combined length of the skull and every

157 cervical, dorsal and sacral centrum (see Hone, 2012 for further details). Since in some cases

158 measurements came from different individuals, we scaled both against the femur of the specimen

159 from which it was measured. This was also carried out for various subsets of the data to test the

160 hypothesis that locomotion patterns of different groups affect tail length. We looked at four

161 broad divisions of dinosaurs based on a general understanding of their locomotion: obligate

162 bipeds (theropods, non-sauropodan sauropodomorphs), obligate quadrupeds (sauropods,

163 thyreophorans), bipeds and facultative bipeds (iguanodontids, hadrosaurs, psittacosaurids)

164 together, and quadrupeds and facultative bipeds together.

165 Incomplete tails were not included in the analyses (with the exception of those reconstructed

166 as described above), as it was considered impossible to ascertain the missing material based on

167 the variations in caudal counts (see below). Even tails that appear to be tapering consistently to a 
168 tip may have some considerable length still missing as seen with Diplodocus, for example.

169 However, in an attempt to maximise the limited available data, we also sourced tails that were

170 incomplete, but considered likely to be close to complete. Such tails can at least be used to

171 demonstrate minimum tail lengths, as an incomplete tail that is as long as or longer than a

172 complete tail still demonstrates a genuine difference (data are provided in the Appendix).

173 The definition of what constitutes a 'nearly' complete tail is necessarily subjective given the

174 limitations of the available information. The intention was to include only those judged to have

175 very few caudals missing and only a very short amount of the tail missing in terms of length. In

176 order to estimate this, we took into account the degree of tapering of the tail, the length of

177 material preserved and the tail lengths of close relatives. For example, not included is the

178 diplodocid sauropod Barosaurus AMNH 6341 (McIntosh, 2005), which has 29 preserved

179 caudals that total over $6 \mathrm{~m}$ in length (against a femur of just $1.4 \mathrm{~m}$ in total length). However, the

180 last preserved caudal in AMNH 6341 is $171 \mathrm{~mm}$ long and, while some sauropods have as few as

18135 caudals (Borsuk-Bialynicka 1977), a large amount of tail is considered likely missing, given

182 the size of the caudals present and the considerably higher number of caudals in other

183 diplodocids (e.g. Gilmore, 1936). Note that the holotype of the small hadrosaur Tethyshadros

184 (Dalla Vecchia, 2009) was incorrectly considered complete in Hone (2012) and so is not in these

185 datasets (though it is included in the section on individual centra lengths).

186 The patterns of individual caudal centrum lengths that make up dinosaurian tails were

187 also analyzed. Here data from the above specimens was supplemented with additional, but

188 incomplete, tails as the analysis looked at changes in individual centra as part of a series, rather

189 than the tail as a whole unit. Note that even tails that can be diagnosed as complete are not

190 always included in the analysis, since either information on individual vertebrae lengths was not 
191 available in the literature or the divisions between the vertebrae could not be reliably measured

192 (e.g. the holotype of Jinfengopteryx - CAGS IG 040801).

193 The data is unevenly distributed with a bias towards small theropods (likely a taphonomic

194 bias from Lagerstaetten deposits) and likely further biased due to high interest in the

195 maniraptoran-avian transition, and with a bias against sauropodomorphs (large animals with

196 numerous tail vertebrae that were seldom buried and preserved as complete specimens). Thus,

197 for the data on snout-sacrum vs complete tail length, there were 16 ornithischians (of which

198 seven were ceratopsians), 12 theropods (of which seven were deinonychosaurs), but only three

199 sauropodomorphs.

200 To test the hypothesis that caudal centrum lengths do not follow a simple decrease in size

201 along the series, we used segmented regression (also known as piecewise or broken stick

202 regression) to identify transitions in centrum length (break points). These length transitions were

203 then compared with the boundary between the muscular and less-muscular parts of the tail (the

204 transition point). Essentially, this approach, where appropriate, fits a series of linear regressions

205 to specific subsets of the data. In some cases, however, a simple linear regression will provide a

206 better fit to the data. Because of some debate over the best way to assess goodness of fit for

207 segmented regressions (e.g., Hall et al., 2013) the analysis was started with a Davies test (Davies,

208 2002) for a non-constant regression parameter in the linear predictor (vertebra number) on

209 centrum size using the R package segmented version 0.4-0.0 (Muggeo, 2003, 2008) implemented

210 in $\mathrm{R}$ version 3.0.3 ( $\mathrm{R}$ core team, 2014). This allows for an independent test of whether a

211 segmented regression is justified, meaning that we were able to use a segmented regression

212 where the data support it and a linear regression where there is no evidence to support a more

213 complex fit. 
214 It is possible that the inevitable variations and slight inconsistencies of collecting data from 215 specimens vs the literature or photographs may affect marginal results. Therefore, we also took 216 one specimen (a hadrosaur - TMP 1998.058.001) as a test case for variation in measurements 217 between first hand observations and photographs. Each caudal centrum length was measured 218 physically 10 times, and the same specimen was then photographed and another 10 replicate 219 measurements obtained from these photographs. Segmented regressions were then fitted in the 220 same way as the rest of the study, to both independently estimate the break points and in 221 particular their congruence to each other, and to estimate the transition point (vertebra 12). 222 Where the Davies test suggests that a segmented regression is appropriate, the next question 223 is how many segments to fit. Candidate models with 1-4 breaks were fitted using segmented and 224 the best model was selected on the basis of the Akaike Information Criterion (Akaike, 1974). In 225 essence, this approach selects the model which best balances explanatory power with simplicity. 226 An upper limit of four breaks was chosen, since some specimens had 20 or fewer vertebrae, 227 limiting the number of breaks that could be plausibly fitted.

228 Models used the default parameters in segmented, with quantiles as the starting points for the 229 iterative breakpoint analysis, but with 50 bootstrap samples and a maximum of 10 iterations.

230 Candidate models that could not be fitted in segmented (usually because of gaps in the data) were 231 discarded.

232 In one case (Dyoplosaurus) the Davies test indicated that a simple linear model was 233 appropriate but visual inspection of the fit indicated that there are in fact two separate groups of 234 vertebrae. The breakpoint models fitted by segmented assume a continuous relationship but in 235 this case it was clear that a discontinuous model would be best. Therefore, one was fitted by 
236 classifying the vertebrae into two groups and then including this as a factor in a linear model plus

237 the interaction term with vertebra number.

238

239

240

241

242

243

244

\section{Results}

\section{Tail length vs snout-sacrum length}

Our hypothesis that there is high variation in tail lengths across the dinosaurs, and that tail lengths do not correlate well with body size (i.e. snout-sacrum length) was supported with very wide confidence limits for predictions of one based on the other (Fig. 2). However, the prediction that there would be similarities between bipedal or quadrupedal taxa was not met. Even when considering facultatively bipedal or quadrupedal taxa with obligate biped and quadrupeds, no clear relationship between body size and tail length was recovered.

The model suggests that tail length is an extremely poor predictor of snout to sacrum length across dinosaurs (Fig. 2) (linear regression of snout/sacrum to femur ratio on tail to femur ratio: $\left.\mathrm{F}_{1,21}=0.11, \mathrm{p}=0.74\right)$. For example, Scutellosaurus has a tail to femur ratio of 8.8 , and with a femur length of $82 \mathrm{~mm}$, this corresponds to an estimated snout-sacrum size between 263.0 and $485.1 \mathrm{~mm}$, though the real value is much closer to the upper bound of $405 \mathrm{~mm}$. At the opposite end of the scale, the tail to femur ratio of 1.2 in Epidexipteryx would correspond to an estimated snout-sacrum range of 173.4 to $259.1 \mathrm{~mm}$ although the actual value is outside of even this broad range at $158 \mathrm{~mm}$.

The same poor prediction of snout-sacrum length by tail length is retained (linear regression: $\mathrm{F} 1,16=0.01, \mathrm{p}=0.91)$ if we attempt to minimise problems due to intraspecific (ontogenetic) scaling or phylogenetic bias (where there are multiple specimens from some species that are therefore over-represented) and restrict the analysis to the largest individual of each species. This pattern is also true of the various subdivisions based on locomotor style: 
260 bipeds alone (linear regression of snout-sacrum to femur vs tail to femur) - $F(1,8)=0.01, p=0.92$;

261 quadrupeds alone $-\mathrm{F}(1,5)=0.2938, \mathrm{p}=0.61$; bipeds plus facultative bipeds $-\mathrm{F}(1,14)=0.331$,

$262 \mathrm{p}=0.57$; quadrupeds plus facultative bipeds $-\mathrm{F}(1,11)=0.009, \mathrm{p}=0.92$. In short, tail size is not 263 clearly related to body size in non-avian dinosaurs, even allowing for broad distinctions in 264 locomotor style.

265

266

267

268

269

270

271

272

273

274

275

276

277

278

279

280

281

282

\section{Patterns of caudal vertebrae length}

Across the Dinosauria, most tail sequences passed the Davies test and so could be reconstructed with one or more breaks to the series of individual centrum lengths. Dinosaur tails do not show simple patterns of change in centrum lengths but typically exhibit multiple distinct regions. There is at least some consistency in the results within clades, with several specimens of single genera showing similar patterns to one another, though others show high levels of variation within one species. The details of these results for various species and clades are considered in more detail below.

\section{Assessment of consistency of centrum measurements}

Where multiple measurements were taken from one hadrosaur tail as a test of consistency of measurements, the congruence was good between break points produced from direct measurements of the specimen and a photograph. In both cases, the model fitted four break points (specimen $($ mean \pm se): $12.0 \pm 1.59,45.8 \pm 3.48,61.5 \pm 3.90,74.7 \pm 0.71$; photographs $11.9 \pm 1.99,48.5 \pm 1.29,57.3 \pm 1.07,59.4 \pm 1.04)$. In particular, both methods fitted a break point very close to the actual transition point, and even at the distal end, where there was slight disagreement, all four of the break points fitted from photographs were encompassed with the 
283 standard errors of break points derived from the specimen itself (Fig. 3). This suggests that the 284 measurements taken from photographs for various specimens will yield accurate data.

285

286 Break points

$287 \quad$ For 18 out of 25 specimens, the distance between a break point and the transition point was 288 lower than would be expected by chance (exact binomial test, $\mathrm{p}=0.043$ ) (Fig. 4). This suggests 289 that there is a relationship between the end of the lateral processes (and by extension the 290 termination of the caudofemoralis muscles) and a change in length of the centra in the tail.

291 The results of the break point analyses are shown in figures 5-12. These are the non292 sauropodan sauropodomorphs (Fig. 5), sauropods (Fig. 6.), earlier branching theropods (Fig. 7), 293 mairaptorans (Fig. 8), dromaeosaurs (Fig. 9), Coelophysis and Archaeopteryx (Fig. 10), 294 thyreophorans (Fig. 11), iguanodontians and hadrosaurs (Fig. 12) and ceratopsians (Fig. 13). 295 Some genera are extremely conservative, such as Archaeopteryx, with others varying 296 considerably, such as Coelophysis (both Fig. 10). Most taxa show an early short series of tail 297 vertebrae that decrease in length sequentially, then a short series that increase in length (typically 298 including the longest centra in the tail), followed by a long series of progressive decrease. These 299 include at least one specimen each of Lufengosaurus (Fig. 5), Apatosaurus, Camarasaurus (both 300 Fig. 6), Gorgosaurus, Tyrannosaurus (both Fig. 7), Ornithomimus, Nomingia (both Fig. 8), 301 Microraptor, and Velociraptor (both Fig. 9), Kentrosaurus (Fig. 11), and Leptoceratops and 302 Centrosaurus (both Fig. 13). Frequently, the first and second caudal vertebrae may vary from 303 this pattern. While not universal, this pattern is widespread in the Dinosauria. Other specimens 304 are close to this pattern (e.g., Dilophosaurus (Fig. 7), Ingenia (Fig. 8), Ouranosaurus and 305 Lambeosaurus (both Fig. 12)) with for example, one extra break point in the series. It is possible 
306 that this pattern is even more prevalent but is, in some instances, hidden from the tests used here

307 by some variation or lack of data. For example, the proximal caudals of Diplodocus and

308 Majungasaurus are not recorded, and although those preserved seem to conform to the pattern, it

309 cannot be confirmed. In the case of Dyoplosaurus the discontinuous model was the best

310 supported (AIC simple linear regression. $=121.7$, AIC discontinuous model $=96.0$ ) and so this

311 was retained (Fig. 11) with a proximal section of short vertebrae with decreasing centrum lengths

312 and a distal segment of much longer, and near stable, centrum lengths.

313

314 Discussion

315 Overall lengths of dinosaurian tails

316 Dinosaur tail lengths vary widely overall and the correlations between tail length and 317 snout-sacrum lengths for complete tails is poor. The data from the 'near complete' tails is of 318 course limited in its use given these issues, although some specimens do suggest that there may

319 be some consistency within groups. For most dinosaur groups, attempting to estimate the total 320 length of a tail from anything other than a near complete series is subject to a wide range of error 321 and uncertainty, as seen even within some clades represented by few specimens (e.g., 322 Scansoripterygidae). Dinosaur tails were also likely evolutionally plastic, given the highly 323 derived forms seen in clades, such as ankylosaurs and diplodocids. Similarly, multiple lineages 324 show adaptation of the tail to specific functions (or specific combinations of functions) such as 325 defense and socio-sexual signaling, adding further to interspecific, and perhaps also intraspecific, 326 variation.

328 Caudal length patterns in dinosaur tails 
329 Considerable variation is seen not just in the overall and proportional sizes of dinosaur tails, but

330 also in the lengths of the individual caudal centra that comprise them. Although the distalmost

331 caudals of a series are generally smaller than more proximal ones, over a short section of

332 consecutive elements, there may be patterns of increasing length, stability, or decreasing length

333 (and all three may occur in one individual e.g., Apatosaurus CM 563 - Gilmore, 1936). The

334 datasets here are somewhat limited, but cover a wide range of dinosaurian biology - large and

335 small, herbivores and carnivores, bipeds and quadrupeds, long and short tails, and taxa from

336 multiple environments. Thus, considerable variation may be expected, but there are nonetheless

337 some clear patterns. Most notably, many dinosaurs show repeated series of, on average,

338 increasing and decreasing centra lengths along the caudal series as demonstrated by the positions

339 of break points and the associated regressions.

340 However, other taxa deviate considerably from the short-long-shortening pattern described

341 above (e.g. Plateosaurus (Fig. 5), Juravenator (Fig. 7)); indeed the caudals of Coelophysis tend

342 to increase in length for much of the series (Fig. 10). Various constraints may confound the basic

343 pattern and affect the overall distribution. For example, the majority of the vertebrae in the

344 dromaeosaurs Velociraptor, Deinonychus and Microraptor are bound by a complex series of

345 extended zygopophyses and chevrons that stiffen the tail and perhaps free the vertebrae from

346 normal functional constraints. This may explain some of the variation seen between specimens of

347 a species or genera (Fig. 10). However, the patterns of centrum lengths seen in the tails of all

348 three specimens of Archaeopteryx and the putative glider Microraptor are strikingly similar and,

349 although only a very limited set of data, show a level of consistency often not seen in other

350 groups (Figs. 9, 10). This suggests that the similarity in form is connected to the shared tail

351 function of control in flight. It has been noted by Gatesy and Dial (1996) that Archaeopteryx 
352 would benefit in flight control from a stiff tail that was only flexible at the very base, and such a

353 condition is reflected here by a proximal section of short centra and then a sudden jump to

354 considerably longer centra.

355 Juravenator is notable as it differs greatly from most other taxa in its pattern of centra

356 lengths (Fig. 7). The specimen is a young juvenile, and it is possible that the tail changes during

357 ontogeny, although Chiappe and Göhlich (2010) noted that the caudal length pattern of

358 Juravenator (a stable series, then a series of short centra, then a series of long centra, and then a

359 series of shorter centra again) may be consistent across at least some compsognathids. This

360 suggests that Juravenator is perhaps not just an outlier, but is representative of a pattern that is

361 normal for its clade. Still, it remains unusual compared to most other dinosaurs.

362

363 Error

364 Given the limited availability of data and the problems associated with sourcing information 365 from the literature, mounted specimens, or those with poor preservation, there is likely to be

366 some error in the data. Distortion, if systematic within a specimen, would still preserve the

367 pattern overall and if random (within or between specimens) should have limited overall effect.

368 This is similar to any issues of measurements taken from the literature where different authors

369 may have used slightly different metrics to take the length of the caudal centra, but we would

370 expect consistency within specimens and thus preservation of patterns. As noted above, our own

371 assessment of the possible differences between the measurements from a specimen and from a

372 photograph produced similar results, especially for the prediction of the transition point which

373 was identical in both and correctly identified as caudal vertebra 12 (Fig. 3). 
The vertebrae of smaller specimens may cause problems, as these will be harder to measure

375

376

377

378

379

380

381

382

383

384

385

386

387

388

389

390

391

392

393

394

395

396

with equivalent accuracy. For example, the long sequence of identical values seen in Juravenator could be because the animal is still a juvenile or may genuinely reflect the unusual caudal anatomy of this genus or of compsognathids as a whole. However, it could simply be because the vertebrae are so small that variation between them (even when attempting to measure to the nearest $0.1 \mathrm{~mm}$ ) was difficult to detect. Similarly, the alternating sequence of long-short-long seen in various specimens of Apatosaurus as described above, may simply be size related (it is easier to measure long vertebrae and find clear differences between them). However, these errors in measurements are collectively likely to be unbiased overall and so should not affect the results or general patterns reported here.

There is some consistency within clades, with for example two specimens of Leptoceratops (despite differing caudal counts - Fig. 13), three specimens of Archaeopteryx (Fig. 10), and two tyrannosaurids (Fig. 7) all showing similar patterns to one another within their respective clades, suggesting consistency in the data and the analysis. Two specimens of Velociraptor are very similar, although a third is rather different, but similar to its near relative Deinonychus (Fig. 9).

\section{Implications}

Assuming equivalency of vertebral articulation, for a given unit of tail length, more joints will increase flexibility and fewer joints will reduce flexiblity. Thus, shorter centra imply greater zonal flexibility and longer ones, greater zonal stiffness. As such, we can use the varying lengths of centra within different parts of dinosaur tails to infer differing levels of zonal flexion.

The repeated pattern of a series of short centra, then a series of long ones, and then a series of tapering caudals suggests that many dinosaurs had a flexible tail base, then a stiffened 
397 section and finally a more flexible section. Our hypothesis that the transition point (i.e. the

398 termination of the attachment of the caudofemoralis musculature) of dinosaurs is linked to a 399 major change in tail function (as implied by centrum length) is supported by our analyses. We 400 recorded the last centrum to preserve a lateral process from specimens directly or where they 401 were recorded in the literature. Comparing these to the data on serial variation shows that the 402 transition point often coincides closely with a shift in centra length, (Fig. 4) as determined by the 403 changes in regression lines (e.g. Lambeosaurus, Fig. 12, Juravenator, Gorgosaurus,

404 Tyrannosaurus, all Fig. 7, Ornithomimus, Fig. 8).

405 Even in taxa where this pattern does not hold, there is evidence that the transition has an 406 influence on caudal length, as the point recorded may be associated with a slight 'hump' in the 407 data (i.e. a short increase and then decrease in vertebral length over just four or five vertebrae), 408 as seen in e.g., Plateosaurus (Fig. 5) and Nomingia (Fig. 8). As the transition point is perhaps 409 better thought of as a 'zone' (Persons \& Currie 2011b), a little leeway must be allowed and we 410 would not expect a perfect correlation between the change in centrum length and the last vertebra 411 possessing a lateral process. Even so, it is clear that the calculated break points in the series often 412 fall exactly on, or within one or two vertebrae of, the last centrum with a lateral process. Based 413 on this, it may be possible to deduce the transition point in some specimens, even if the lateral 414 processes and chevrons are missing or damaged, by interpreting the pattern of centrum lengths. 415 The series of long vertebra that correspond to most of the length of the anterior tail up to the 416 transition point would make the tail relatively stiff, since, in the absence of other anatomical 417 changes, longer vertebra lead to a reduction in intervertebral flexure points per unit of length. 418 This would improve the efficiency of the caudofemoralis muscles as this stiffness would reduce 
419 energy loss though lateral movements between vertebrae and ensure that most of the effort of 420 contracting the muscles led to pull on the femur and not lateral tail flexing.

421 Posterior to the transition point, the requirement for great lateral stiffness is presumably

422 relaxed and thus leads to the simple pattern of general reduction in size of successive centra 423 moving posteriorly. Many taxa independently evolved post-transition-point adaptations for 424 increasing caudal stiffening, such as the relatively elongated centra and extensive zygopophyses 425 of tetanurans, the caudal rods of dromaeosaurs, the pygostyles of various maniraptorans and the 426 handle of ankylosaur tail clubs. Simple selection for reduction in mass posteriorly (with obvious 427 exceptions such as taxa bearing tail clubs) would lead to the pattern seen here and may be the 428 typical primitive condition for reptilian tails. That is seen here in the data for Varanus and 429 Crocodylus (SI Fig. 4), and this pattern was also recorded from some other extant and extinct 430 reptiles, and even synapsids by Nuñez Demarco et al. (2018), suggesting that it is a common 431 feature of early amniotes (though clearly this is a very limited dataset).

432 We suggest that the series of short centra at the base of the tail would allow the entire tail to 433 flex as a unit, as any anterior motion of the tail would also affect any more posterior portion of 434 the tail. Thus a flexible section at the very base of the tail allows the entire tail to be moved 435 without compromising the stiffness of the successive section. This is therefore likely a trade-off 436 between flexion and stiffness. The most proximal section of the tail may also have been less 437 strongly influenced by contractions of the caudofemoral musculature, because the most proximal 438 caudal vertebrae typically lack chevrons, which contribute to the origin of the caudofemoralis. 439 This would afford far less muscle attachment than in the stiffened, middle section of the tail. 440 Additionally, the bending moment of the caudofemoralis would be proportional to the small sine 
441 of the angle between the origins of the caudofemoralis and its lines of action, making the most

442 anterior vertebrae less strongly influenced by this action.

443

444 Evolution of dinosaurian caudal centra series

445 The short-long-decreasing pattern was likely acquired independently in multiple lineages of

446 dinosaurs. We hypothesise this based on the number of Triassic and / or basal forms

447 (Plateosaurus, Fig. 5; Coelophysis, Fig. 10), that lack the pattern and present the apparently

448 primitive archosaur (or even diapsid) condition of a simple progressive decrease along the length

449 of the tail. The repeated evolution of various osteological structures able to passively stiffen the

450 tail (e.g. elongate zygopophyses and chevrons in some theropods and ankylosaurs, hyposphene-

451 hypantra articulations in some sauropods and alvarezsaurs, ossified tendons in ornithischians)

452 suggests that tail rigidity was favoured repeatedly in various groups within the Theropoda,

453 Sauropoda and Ornithischia. Thus, the apparent distribution of the short-long-decreasing pattern

454 seen here in later theropods, sauropods and ornithischians may have also arisen independently

455 from similar selective pressures favouring a small zone of high flexibility immediately posterior

456 to the hips and an extended zone of stiffness that helped improve locomotor efficiency.

457 Despite the wide variations in patterns of elongation and constriction in centrum lengths,

458 it is clear that in at least some cases where there is a reasonable number of caudals preserved and

459 their positions known, it may be possible to reconstruct the size of missing vertebrae with some

460 confidence. Repeated patterns within and between taxa, and long strings of caudals with a

461 consistent pattern of elongation or reduction means that the sizes of missing vertebrae may be

462 estimated. Potentially, even the total length of a tail may be estimated if much of the series is

463 preserved. However, in general this is likely to be difficult - the variation seen here in the 
464 patterns of increases and decreases, and the differing numbers of vertebrae in those various sets 465 of increases and decreases are highly variable and difficult to predict. In particular, guessing at 466 what number of vertebrae a tail will end is difficult. While clearly any regression of caudal size 467 that was decreasing successively would eventually suggest a centrum of near zero or negative 468 length, at what point before this the tail would actually terminate cannot be estimated. Gilmore 469 (1936) notes with relation to the 'whiplash' segment of the distal tail of Apatosaurus that "the 470 uniformity in size of these terminal rod-like caudals is such that any loss would be difficult to 471 detect”. Similarly, pygostyles of some theropods (which can reduce very rapidly in size along 472 their length) could also be misleading, as, if only the proximal part were preserved, it would 473 erroneously suggest a much longer tail.

474 Recognizing variation in caudal counts across taxa has implications for other areas of 475 research. For example, knowing how many vertebrae and of what size ranges a given taxon has 476 may be important to taphonomic analyses that consider sorting or loss of elements by size. In an 477 analysis of a bone bed dominated by the hadrosaur Amurosaurus, Lauters et al. (2008) looked at 478 the different numbers of element types preserved. They suggested that the vertebrae of 479 Amurosaurus were underrepresented in the bonebeds based on the estimated number of vertebrae 480 in the axial column. However, with very little articulation known for remains of Amurosaurus, it 481 cannot be easily estimated how long the tail was or how many caudals it possessed, with tails for 482 hadrosaurs known to have as few as around 50 caudals (Horner, Weishampel \& Forster, 2004) to 483 over 75 (Lull \& Wright, 1942). (There is of course also likely variation within the number of the 484 cervical, dorsal and sacral series, though based on Hone [2012], this is likely to be much less of 485 an issue than the caudal series). The results of Lauters et al. (2008) for Amurosaurus, were robust 486 and in this case such an issue is not likely to have had a major effect on their results, but the 
487 488 489 490 491 492

\section{3} 494 495 496 497 498 499

500 501 502 503

504

505

506 508 509 510

507 Future studies of variation patterns in other traits (such as centrum width and zygapophyseal

uncertainty surrounding the number of vertebrae in the axial column means that care should be taken when performing such an analysis. We suggest that, unless the true axial count is known with confidence, either caudals should not be counted, or upper and lower estimates of the number of vertebrae in the column should be employed, or such considerations should be limited to the lateral process bearing caudals.

\section{Conclusions}

Total tail length remains difficult to estimate for incomplete tails in the Dinosauria, and there is strong variation both between and within Family rank equivalent clades. However, there is some consistency in patterns of individual centra length within tails. Notably, the proximal part of the tail often consists of relatively short vertebrae, followed by a series of longer vertebrae and then a shift to decreasing centrum lengths beyond the transition point. This pattern suggests an underlying constraint in dinosaurian tail function, where (1) the very base of the tail was flexible and allowed the large remaining posterior portion of the tail to be swung as a collective whole, (2) shortly past the tail base there was a zone of relative stiffness that supported the caudofemoralis musculature, and (3) after the termination of the caudofemoralis and for a highly variable distance, the remaining vertebrae tapered to a reduced size.

While length of centrum is the most relevant measure of an individual vertebrate's contribution to total tail length, numerous other traits have the potential to influence basic aspects of caudal function, including lateral flexibility and the functioning of the caudofemoralis. articulation) will refine these general observations. Although developed in order to investigate dinosaur tails, the methods used here to separate out groups of similar units as part of a series may be widely applicable to assessments of any extinct and extant taxa exhibiting repeating 
511 anatomical units (e.g. the dimensions of any vertebral series, scalation, or body segments in

512 annelid or arthropod invertebrates).

513

\section{Acknowledgements}

515

516 We wish to thank the numerous colleagues who provided papers, discussions, and details of 517 specimens with complete tails that are buried in the literature and research collections: Jordan

518 Mallon, Matthew Herne, Susie Maidment, Corwin Sullivan, Fabio Dalla Vecchia, Andrea Cau, 519 Victoria Arbour, Mickey Mortimer, Phil Currie, Matt Wedel, Pascal Godefroit and Chris Rogers.

520 For access to specimens we thank Sandra Chapman and Fang Zheng. We thank Mike Habib for 521 discussion of tail evolution and stiffening and Rob Knell for assistance with the statistics. We 522 also thank Victoria Arbour, Heinrich Mallison, Eric Snively, Susie Maidment and David Polly 523 for comments on an earlier version of this manuscript and Mark Young for handling it as editor.

524

525

526

527

528

529

530

531

532

533

534 535

\section{References}

Akaike H. 1974. A new look at the statistical model identification. IEEE Transaction of Automatatic Control 19:716-23.

Allen V, Paxton H, Hutchinson JR. 2009. Variation in center of mass estimates for extant sauropsids and its importance for reconstructing inertial properties of extinct archosaurs. Anatomical Record 292:1442-1461.

Alroy J. 2019. Small mammals have big tails in the tropics. Global Ecology and Biogeography, 28:1042-1050.

Arbour VM. 2009. Estimating impact forces of tail club strikes by ankylosaurid dinosaurs. PLoS ONE 4:e6738. 
536 Borsuk-Bianka M. 1977. A new camarasaurid sauropd Opisthocoelicaudia skarzynskii, gen. n.

537 sp. n. from the Upper Cretaceous of Mongolia. Acta Palaeontologica Polonica 37:5-64.

538 Campione NE, Evans DC. 2012. A universal scaling relationship between body mass and

539 proximal limb bone dimensions in quadrupedal terrestrial tetrapods. BMC biology 10:60.

540 Cau A, Serventi P. 2017. Origin attachments of the caudofemoralis longus muscle in the Jurassic

541 dinosaur Allosaurus. Acta Palaeontologica Polonica 62:273-277.

542 Cavallini, P. 1995. Variation in the body size of the red fox. Annales Zoologici Fennici 32:421-

$543 \quad 427$.

544 Chiappe LM, Göhlich UB. 2010. Anatomy of Juravenator starki (Theropoda: Coelurosauria)

545 from the Late Jurassic of Germany. Neues Jahrbuch für Geologie und Paläontologie-

$546 \quad$ Abhandlungen 258:257-296.

547 Currie PJ. 1978. The orthometric linear unit. Journal of Paleontology 52: 964-971.

548 Dalla Vecchia F. 2009. Tethyshadros insularis, a new hadrosauroid dinosaur (Ornithischia) from

549 the Upper Cretaceous of Italy. Journal of Vertebrate Paleontology 29:1100-1116.

550 Davies RB. 2002. Hypothesis testing when a nuisance parameter is present only under the

$551 \quad$ alternative: linear model case. Biometrika 89:484-489.

552 Garland T Jr. 1985. Ontogenetic and individual variation in size, shape and speed in the

553 Australian agamid lizard Amphibolurus nuchalis. Journal of Zoology 207:425-439.

554 Gauthier JA. 1986. Saurischian monophyly and the origin of birds. Memoirs of the California

555 Academy of Sciences 8:1-55.

556 Gatesy SM. 1990. Caudefemoral musculature and the evolution of theropod locomotion.

$557 \quad$ Paleobiology 16:170-186. 
558 Gatesy SM, Dial KP. 1996. From frond to fan: Archaeopteryx and the evolution of short-tailed $559 \quad$ birds. Evolution 50:2037-2048.

560 Gatesy SM, Thomason JJ. Functional morphology in vertebrate paleontology. Functional $561 \quad$ evolution of the hindlimb and tail from basal theropods to birds. 1995:219-34.

562 Gilmore CW. 1936. Osteology of Apatosaurus, with special reference to specimens in the $563 \quad$ Carnegie Museum. Memoirs of the Carnegie Museum 11:175-271.

564 Hall AR, Osborn DR, Sakkas N. 2013. Inference on Structural Breaks using Information Criteria. 565 The Manchester school of economic and social studies 81:54-81.

566 Head JJ, David Polly P. Dissociation of somatic growth from segmentation drives gigantism in 567 snakes. Biology Letters 22:296-8.

568 Hone DWE. 2012. Variation in the tail length of non-avian dinosaurs. Journal of Vertebrate 569 Paleontology 32:1082-1089.

570 Horner JR, Weishampel DB, Forster CA. 2004. Hadrosauridae. In: Weishampel DB, Dodson P, 571 Osmólska H, eds. The Dinosauria. Berkeley: University of California Press, 438-465.

572 Hutchinson JR, Gatesy SM. 2001. Bipedalism. Encyclopedia of Life Sciences 1-6.

573 Hutchinson JR, Ng-Thow-Hing V, Anderson FC. 2007. A 3D interactive method for estimating 574 body segmental parameters in animals: application to the turning and running 575 performance of Tyrannosaurus rex. Journal of Theoretical Biology 246:660-680.

576 Lauters P, Bolotsky YL, Van Itterbeeck J, Godefroit P. 2008. Taphonomy and age profile of a 577 Latest Cretaceous dinosaur bone bed in Far Eastern Russia. Palaios 23:153-162.

578 Libby T, Moore TY, Chang-Siu E, Li D, Cohen DJ, Jusufi A, Full RJ. 2012. Tail-assisted pitch 579 control in lizards, robots and dinosaurs. Nature 481:181-184. 
580 Lull RS, Wright NE. 1942. Hadrosaurian dinosaurs of North America. Geological Society of $581 \quad$ America Special Papers 40.

582 Mallison H. 2011. Defense capabilities of Kentrosaurus aethiopicus Hennig, 1915.

$583 \quad$ Palaeontologia Electronica 14:1-25.

584 McIntosh JS. 2005. The genus Barosaurus Marsh (Sauropoda, Diplodocidae). In Tidwell V, 585 Carpenter K, eds. Thunder-lizards: the sauropodomorph dinosaurs Bloomington: 586 Indiana University Press, 8-77.

587 Muggego VMR. 2003. Estimating regression models with unknown break-points. Statistics in $588 \quad$ Medicine 22:3055-3071.

589 Muggego VMR. 2008. segmented: an R Package to Fit Regression Models with Broken-Line 590 Relationships. R News 8:20-25. URL http://cran.r-project.org/doc/Rnews/.

591 Nuñez Demarco P, Meneghel M, Laurin M, Piñeiro G. 2018. Was Mesosaurus a fully aquatic $592 \quad$ reptile?. Frontiers in Ecology and Evolution 6:p.109.

593 Persons WS, Currie PJ. 2011a. The tail of Tyrannosaurus: reassessing the size and locomotive 594 importance of the M. caudofemoralis in non-avian theropods. The Anatomical Record $595 \quad 294: 119-131$.

596 Persons WS, Currie PJ. 2011b. Dinosaur speed demon: the caudal musculature of Carnotaurus 597 sastrei and implications for the evolution of South American abelisaurids. PLOS ONE 598 6:e25763.

599 Persons WS, Currie PJ. 2012. Dragon tails: convergent caudal morphology in winged archosaurs. 600 Acta Geologica Sinica (English edition) 86:1402-1412. 
601 Persons WS, Currie PJ. 2014. Duckbills on the run, the cursorial abilities of hadrosaurs and 602 implications for tyrannosaur avoidance strategies. In: Eberth DA, Evans DC eds. 603 Hadrosaurs. Indiana University Press, Bloomington, Indiana. 449-458.

604 Persons WS, Currie PJ, Norrell MA. 2014. Oviraptorosaur tail forms and functions. Acta 605 Palaeontologica Polonica 59:553-567.

606 Pittman M, Gatesy SM, Upchurch P, Goswami A, Hutchinson JR. 2013. Shake a tail feather: the 607 evolution of the theropod tail into a stiff aerodynamic surface. PLoS ONE 8:e63115.

608 R Core Team. 2014. R: A language and environment for statistical computing. R Foundation for 609 Statistical Computing, Vienna, Austria. URL http://www.R-project.org/

610 Rothschild BM, Depalma RA, Burnham DA, Martin L. 2020. Anatomy of a dinosaur611 Clarification of vertebrae in vertebrate anatomy. Anatomia, Histologia, Embryologia.

612 Russell DA. Ostrich dinosaurs from the Late Cretaceous of western Canada. Canadian Journal 613 of Earth Sciences 9:375-402.

614 Sereno PC. 1987. The ornithischian dinosaur Psittacosaurus from the Lower Cretaceous of Asia 615 and the relationships of the Ceratopsia. Ph.D. Dissertation, Columbia University, New

616 York, $554 \mathrm{pp}$.

617 Wake DB. 1979. The endoskeleton: the comparative anatomy of the vertebral column and ribs. 618 Hyman's comparative vertebrate anatomy. 192:237.

619

620

\section{Figure captions}

622 
623 Figure 1. Example tail forms across the Dinosauria: A, ceratopsid (Centrosaurus nasicornis); B,

624 hadrosaurid (Corythosaurus intermedius); C, anklosaurid (Euoplocephalus tutus); D, diplodocid

625 (Diplodocus carnegii); E, tyrannosaurid (Gorgosaurus libratus). Scale bar is 1 meter.

626

627 Figure 2. Relative size of snout to sacrum against relative tail length. Snout to sacrum vs tail

628 length, with both measurements scaled to femur size (see Methods). Comparisons drawn from

629 the same individual are shown as black circles; those from different individuals as open circles.

630 The solid line shows the fitted (non-significant) regression; dashed lines show 95\% confidence

631 intervals for this regression. The grey lines show the range and distribution of tail to femur ratio

632 for all of the species in our analysis and demonstrate that while most cluster in the centre, there

633 are extremes (from 1.3 to 12.4 ) and here the confidence intervals would be even wider. See also

634 SI Table 1.

635

636 Figure 3. Segmented regressions for the indeterminate hadrosaur specimen TMP1998.058.001.

637 Segments derived from photographs (red) and from the specimen itself (blue). Both methods

638 fitted a break point very close to the actual transition point, and with the exception of a break

639 point right at the distal end, break points derived from one method overlapped with break points

640 derived from the other. The predicted break points and their error bars are indicated at the bottom

641 of each graph where these have been calculated, and the transition point (where known) is

642 indicated by an arrow.

643

644 Figure 4. Mean distance between break point and transition point in 1000 randomisations, plotted

645 against the actual distance. The grey line shows the 1:1 line; red lines show standard errors. For 
646 all but seven of the specimens, the actual distance was lower than the randomised distance (Exact

647 binomial test, $\mathrm{p}=0.04$ ). Abbreviations for listed specimens are as follows: Ap Apatosaurus, Cam

648 Camarasaurus, Cen Centrosaurus, Coe Coelophysis, Dil Dilophosaurus, Dip Diplodocus, Gor

649 Gorgosaurus, Ig Iguanodon, Jur Juraventor, Ken Kentrosaurus, Lam Lambeosaurus, Lep

650 Leptoceratops, Maj Majungasaurus, Mic Microraptor, Nom Nomingia, Ome Omeisaurus, Opi

651 Opistocoelocaudia, Orn Ornithomimus, Our Ouranosaurus, Pla Plateosaurus, Ps Psittacosaurus

652 (1= AMNH 6253, 2 = AMNH 6254), Ten Tenontosaurus, Ty Tyrannosaurus, Vel Velociraptor 653

654 Figure 5. Regressions for centrum lengths within the tails for non-sauropodan members of the 655 Sauropodomorpha, A) Lufengosaurus, B) Plateosaurus. While different patterns are recovered, 656 both show long sections of the tail with a tapering pattern of centra lengths. The predicted break 657 points and their error bars are indicated at the bottom of each graph where these have been 658 calculated, and the transition point (where known) is indicated by an arrow.

659

660 Figure 6. Regressions for centrum lengths within the tails of members of Sauropoda. The 661 predicted break points and their error bars are indicated at the bottom of each graph where these 662 have been calculated, and the transition point (where known) is indicated by an arrow (the same 663 point is inferred in Apatosaurus E based on D). A) Omeisaurus, B) Opsitocoelocaudia, C) 664 Diplodocus, D) Apatosaurus CM3018, E) Apatosaurus CM3378, F) Camarasaurus.

665

666 Figure 7. Regressions for centrum lengths within the tails for non-maniraptoran members of the 667 Theropoda, A), Dilophosaurus, B) Ceratosaurus, C) Majungasaurus, D) Juravenator, E)

668 Gorgosaurus, F) Tyrannosaurus. Note that patterns of the two tyrannosaurs, Gorgosaurus and 
669 Tyrannosaurus, are most similar to each other and that the pattern of Juravenator is the most 670 dissimilar from all other theropods. The predicted break points and their error bars are indicated

671 at the bottom of each graph where these have been calculated, and the transition point (where 672 known) is indicated by an arrow.

673

674 Figure 8. Regressions for centrum lengths within the tails for selected members of non-paravian 675 Maniraptora A) Ornithomimus B) Ingenia, C) Nomingia. The two oviraptorosaurs (B, C) show 676 strongly tapering tails. The predicted break points and their error bars are indicated at the bottom 677 of each graph where these have been calculated, and the transition point (where known) is 678 indicated by an arrow.

679

680 Figure 9. Regressions for centrum lengths within the tails for members of the Dromaeosauridae,

681 A) Velociraptor IGM 100/25, B) Velociraptor AMNH 100-986, C) Velociraptor MPC 100/985), 682 D) Deinonychus, E) Microraptor. The predicted break points and their error bars are indicated at 683 the bottom of each graph where these have been calculated, and the transition point (where 684 known) is indicated by an arrow.

685

686 Figure 10. Regressions for centrum lengths within the tails for specimens of Archaeopteryx (A) 687 BSPG1999I50, B) WDC CGS100 C) $11^{\text {th }}$ Specimen) and Coelophysis (D) AMNH 7223, E) AMNH 7224, F) AMNH7229). Archaeopteryx is a rare genus for which numerous specimens with complete tails are known. Although the general pattern observed across the specimens is

690 similar, the pattern of each specimen is distinct. Despite all three Coelophysis specimens coming 691 from the same locality, their tails differ markedly from each other. The predicted break points 
692 and their error bars are indicated at the bottom of each graph where these have been calculated, 693 and the transition point (where known) is indicated by an arrow.

694

695 Figure 11. Regressions for centrum lengths within the tails for members of the Thyreophora, A)

696 Kentrosaurus, B) Dyoplosaurus. More than any other taxa in the study, the club bearing tail

697 Dyoplosaurus would be expected to show a specialized pattern, and it does, although

698 surprisingly the analysis did not recover break points in the series. The predicted break points

699 and their error bars are indicated at the bottom of each graph where these have been calculated,

700 and the transition point (where known) is indicated by an arrow.

701

702 Figure 12. Regressions for centrum lengths within the tails for members selected non-

703 hadrosauroid iguanodontians, A) Tenontosaurus, B) Ouranosaurus, C) Iguanodon) and

704 hadrosaurs, D) Tethyshadros, E) Lambeosaurus). Despite their overall similarity in form and

705 ecology there are considerable differences between the patterns seen between these taxa. The

706 predicted break points and their error bars are indicated at the bottom of each graph where these

707 have been calculated, and the transition point (where known) is indicated by an arrow.

708

709 Figure 13. Regressions for centrum lengths within the tails for members of the Ceratopsia. A)

710 Psittacosaurus AMNH 6253, B) Psittacosaurus AMNH 6254, C) Psittacosaurus GiSPS

711 100/606, D) Leptoceratops CMN 8887, E) Leptoceratops CMN 8888), E) Centrosaurus.

712 Relative to that of most other dinosaur groups considered, the ceratopsians pattern is closer to

713 simple progressive tapering. The predicted break points and their error bars are indicated at the 
714 bottom of each graph where these have been calculated, and the transition point (where known)

715 is indicated by an arrow.

716

717

718

719

720

721 APPENDICES:

722 Table 1. All data - Complete tail lengths, Snout-Sacrum lengths, Incomplete tail lengths,

723 Centrum lengths, Transverse Processes, References.

724

725 SI Fig 1. Break points for ornithischian tails. Aligned caudal centra (black squares, spaces

726 indicate missing vertebrae), break points as calculated (red points with error bars) and transition

727 point (where known, blue triangles) for all ornithischians in the study.

728

729 SI Fig 2. Break points for sauropodomorph tails. Aligned caudal centra, break points as

730 calculated and transition point (where known) for all sauropodomorphs in the study.

731

732 SI Fig 3. Break points for theropod tails. Aligned caudal centra, break points as calculated and 733 transition point (where known) for all theropods in the study.

734

735 SI Fig 4. Regressions for centrum lengths within the tails for two non-dinosaurian reptiles.

736 Centrum length patterns for Crocodylus niloticus and Varanus nilitocus. Both display a simpler 
737 pattern than that typical of the considered dinosaurs with a relatively consistent decrease in

738 centrum length along the caudal series. The transition point (where known) is indicated by an 739 arrow.

740 


\section{Figure 1}

Figure 1. Example tail forms across the Dinosauria
A) anklosaurid (Euoplocephalus tutus), B) ceratopsid (Centrosaurus nasicornis), C) hadrosaurid (Corythosaurus intermedius), D) diplodocid (Diplodocus carnegii), E) tyrannosaurid (Gorgosaurus libratus). Scale bar is 1 meter. 


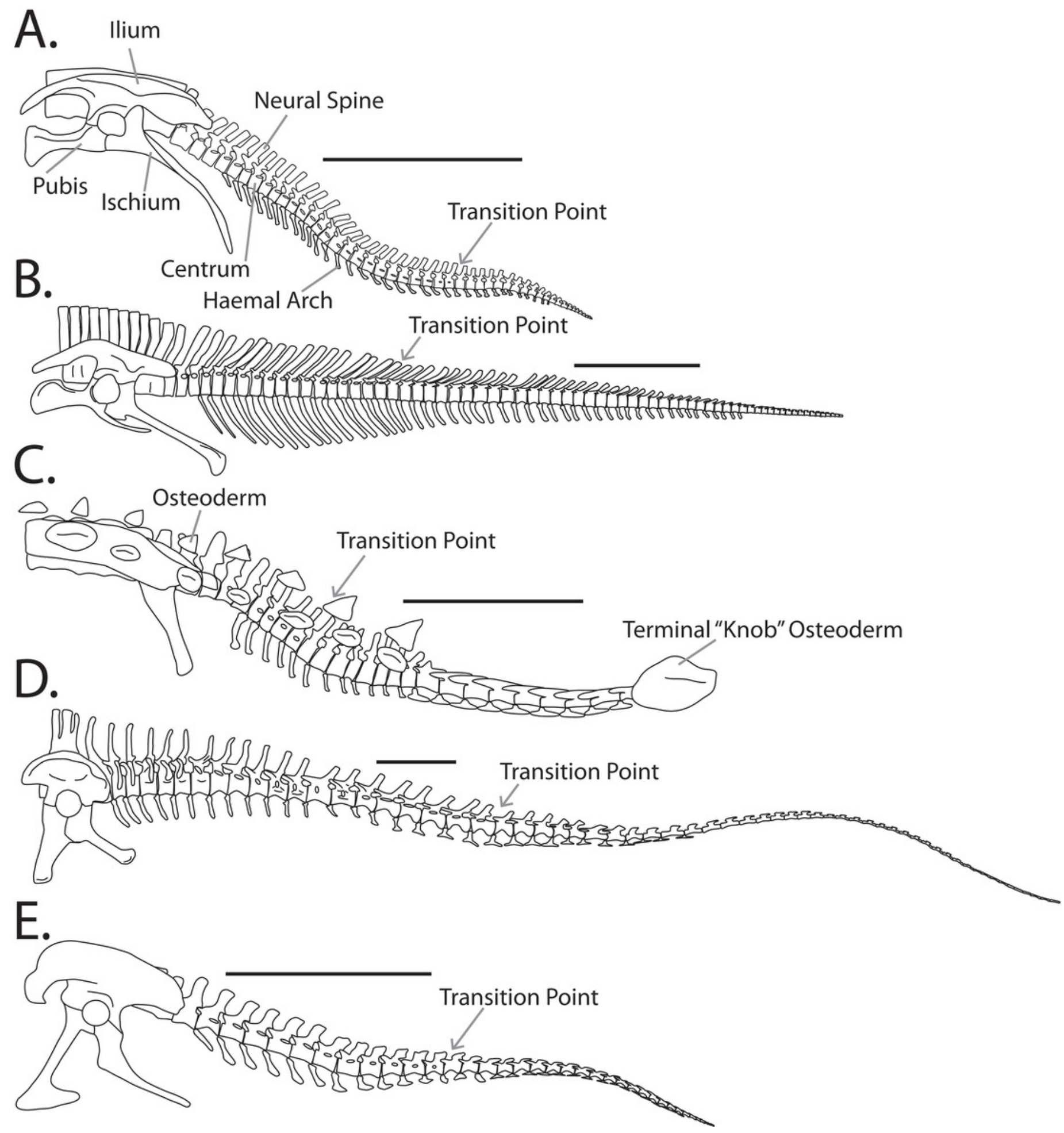




\section{Figure 2}

Figure 2. Relative size of snout to sacrum against relative tail length.

Snout to sacrum vs tail length, with both measurements scaled to femur size (see Methods).

Comparisons drawn from the same individual are shown as black circles; those from different individuals as open circles. The solid line shows the fitted (non-significant) regression; dashed lines show $95 \%$ confidence intervals for this regression. The grey lines show the range and distribution of tail to femur ratio for all of the species in our analysis and demonstrate that while most cluster in the centre, there are extremes (from 1.3 to 12.4) and here the confidence intervals would be even wider. See also SI Table 1. 


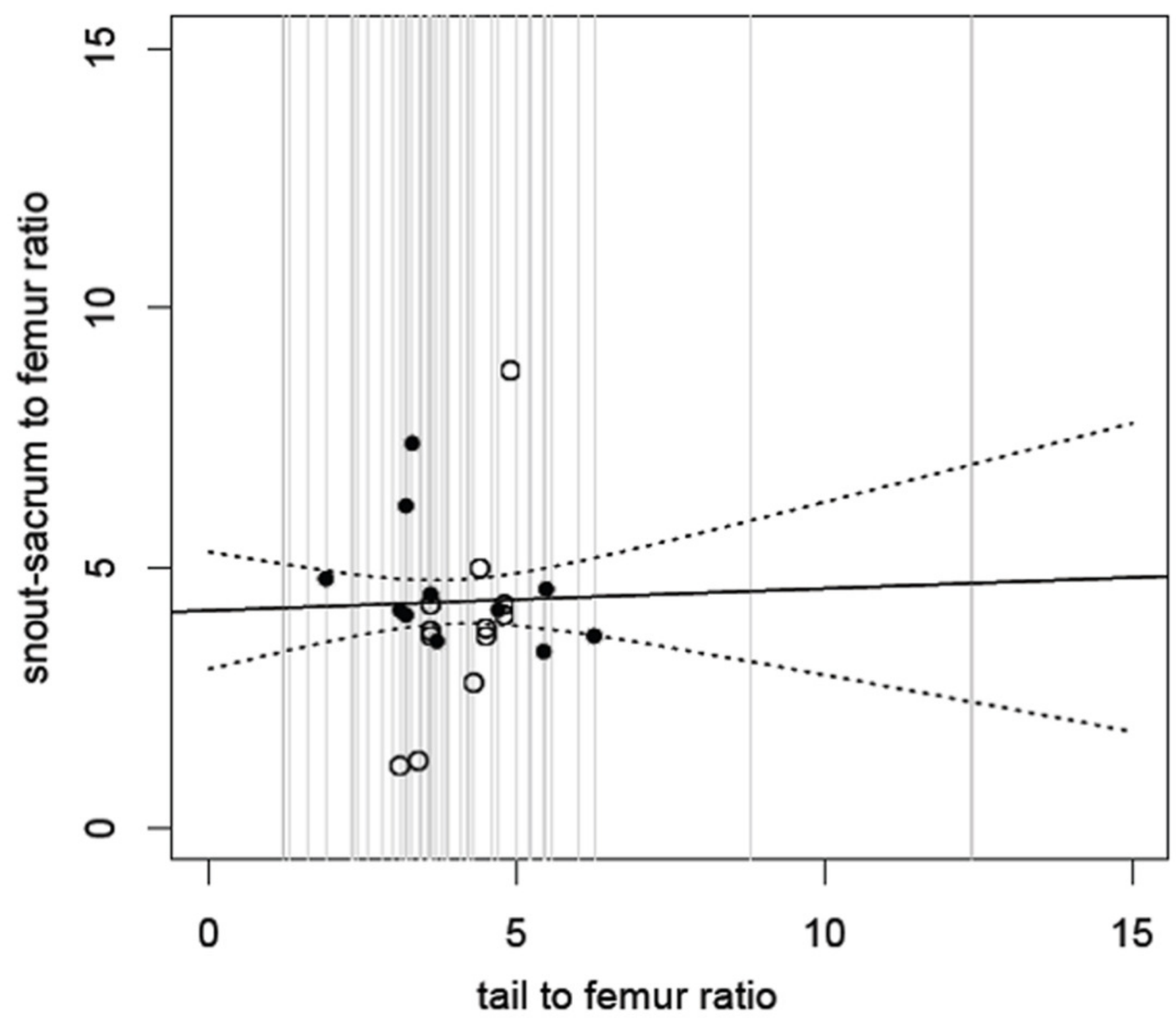




\section{Figure 3}

Figure 3. Segmented regressions for the indeterminate hadrosaur specimen TMP1998.058.001.

Segments derived from photographs (red) and from the specimen itself (blue). Both methods fitted a break point very close to the actual transition point, and with the exception of a break point right at the distal end, break points derived from one method overlapped with break points derived from the other. The predicted break points and their error bars are indicated at the bottom of each graph where these have been calculated, and the transition point (where known) is indicated by an arrow. 


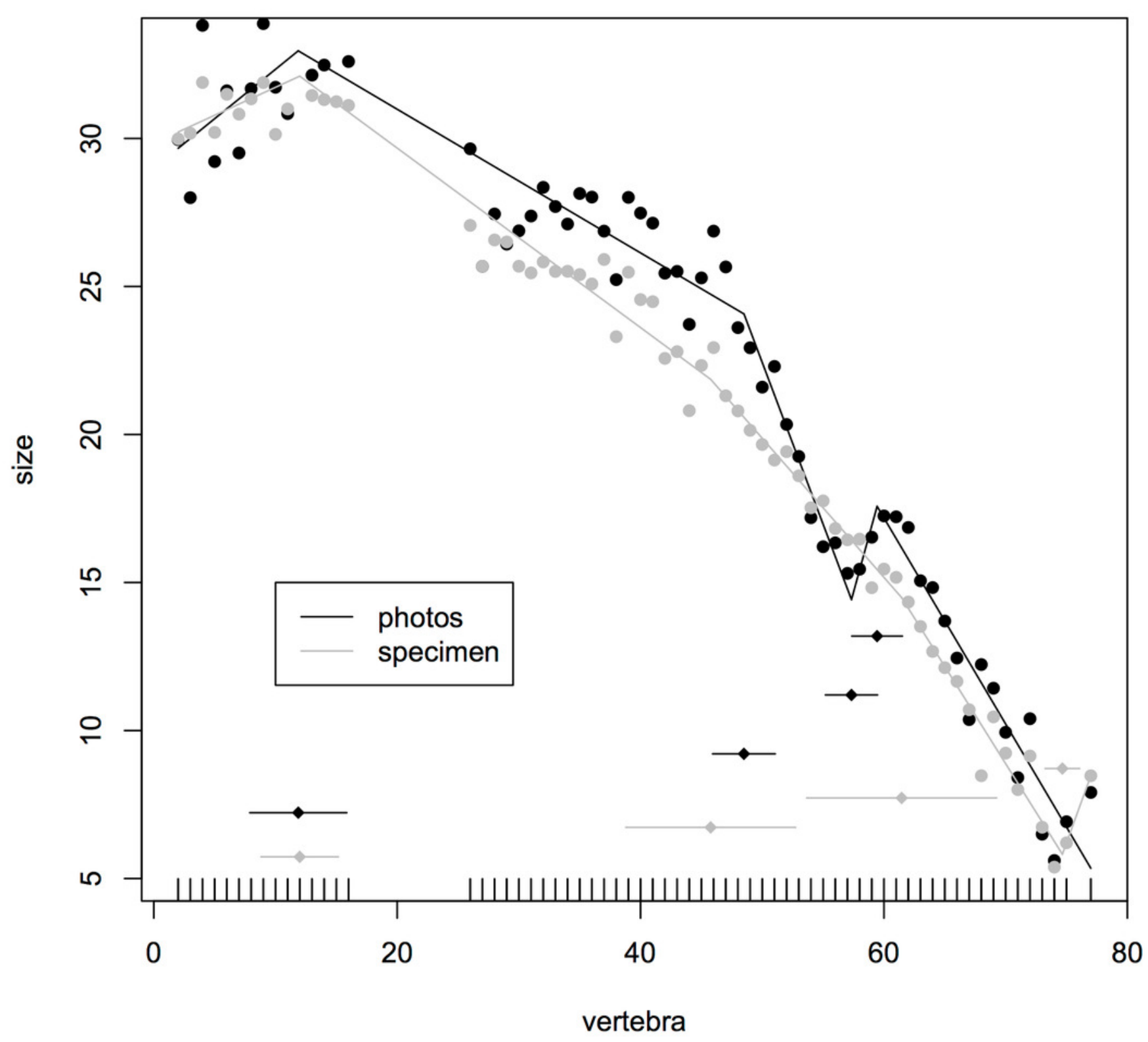




\section{Figure 4}

Figure 4. Mean distance between break point and transition point in 1000 randomisations, plotted against the actual distance.

The grey line shows the 1:1 line; red lines show standard errors. For all but seven of the specimens, the actual distance was lower than the randomised distance (Exact binomial test, $p=0.04)$. Abbreviations for listed specimens are as follows: Ap Apatosaurus, Cam Camarasaurus, Cen Centrosaurus, Coe Coelophysis, Dil Dilophosaurus, Dip Diplodocus, Gor Gorgosaurus, Ig Iguanodon, Jur Juraventor, Ken Kentrosaurus, Lam Lambeosaurus, Lep Leptoceratops, Maj Majungasaurus, Mic Microraptor, Nom Nomingia, Ome Omeisaurus, Opi Opistocoelocaudia, Orn Ornithomimus, Our Ouranosaurus, Pla Plateosaurus, Ps Psittacosaurus (1= AMNH 6253, 2 = AMNH 6254), Ten Tenontosaurus, Ty Tyrannosaurus, Vel Velociraptor 


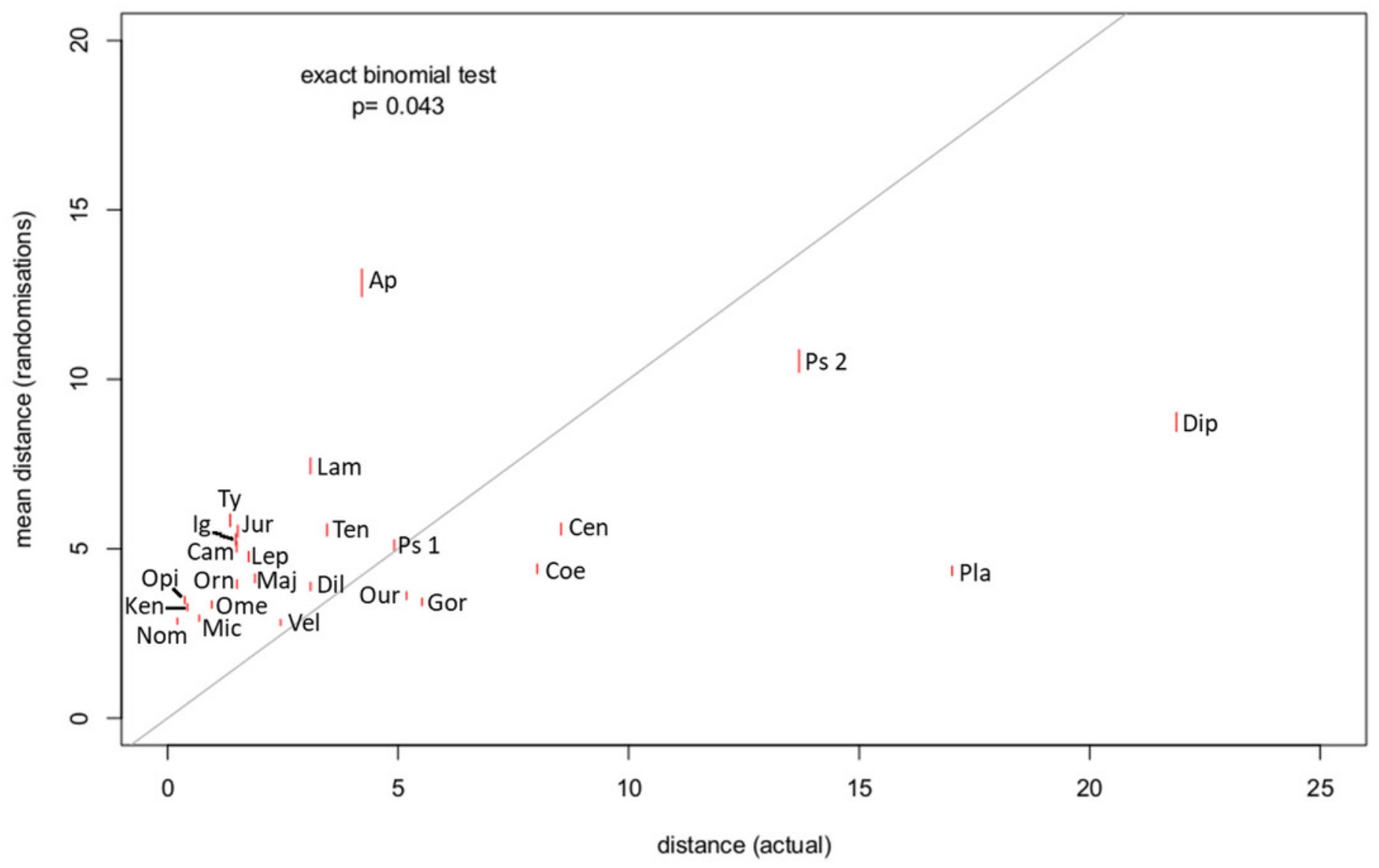




\section{Figure 5}

Figure 5. Regressions for centrum lengths within the tails for non-sauropodan members of the Sauropodomorpha, A) Lufengosaurus, B) Plateosaurus.

While different patterns are recovered, both show long sections of the tail with a tapering pattern of centra lengths. The predicted break points and their error bars are indicated at the bottom of each graph where these have been calculated, and the transition point (where known) is indicated by an arrow. 

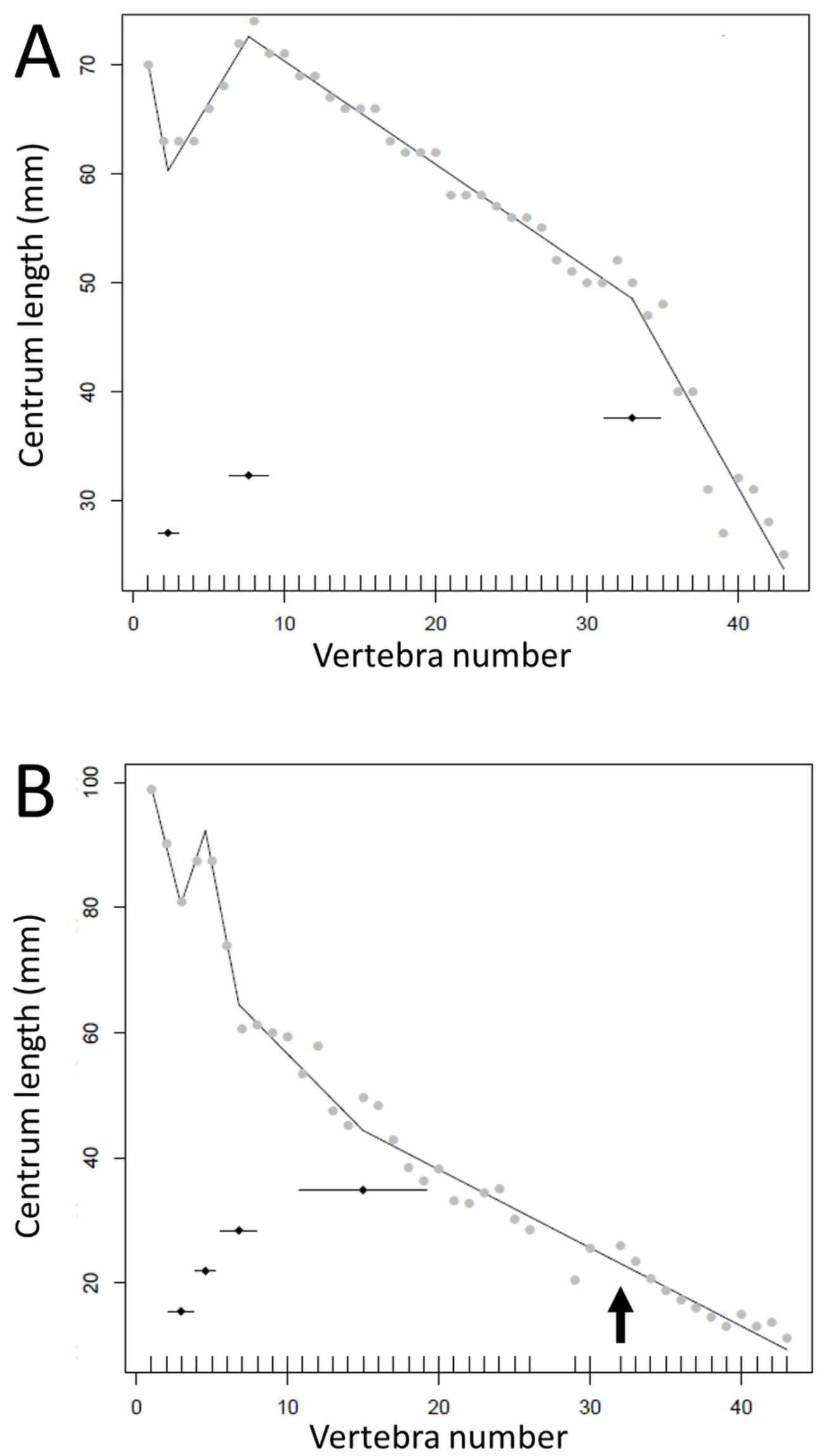


\section{Figure 6}

Figure 6. Regressions for centrum lengths within the tails of members of Sauropoda.

The predicted break points and their error bars are indicated the bottom of each graph where these have been calculated, and the transition point (where known) is indicated by an arrow (the same point is inferred in Apatosaurus E based on D). A) Omeisaurus, B) Opsitocoelocaudia, C) Diplodocus, D) Apatosaurus CM3018, E) Apatosaurus CM3378, F) Camarasaurus. 

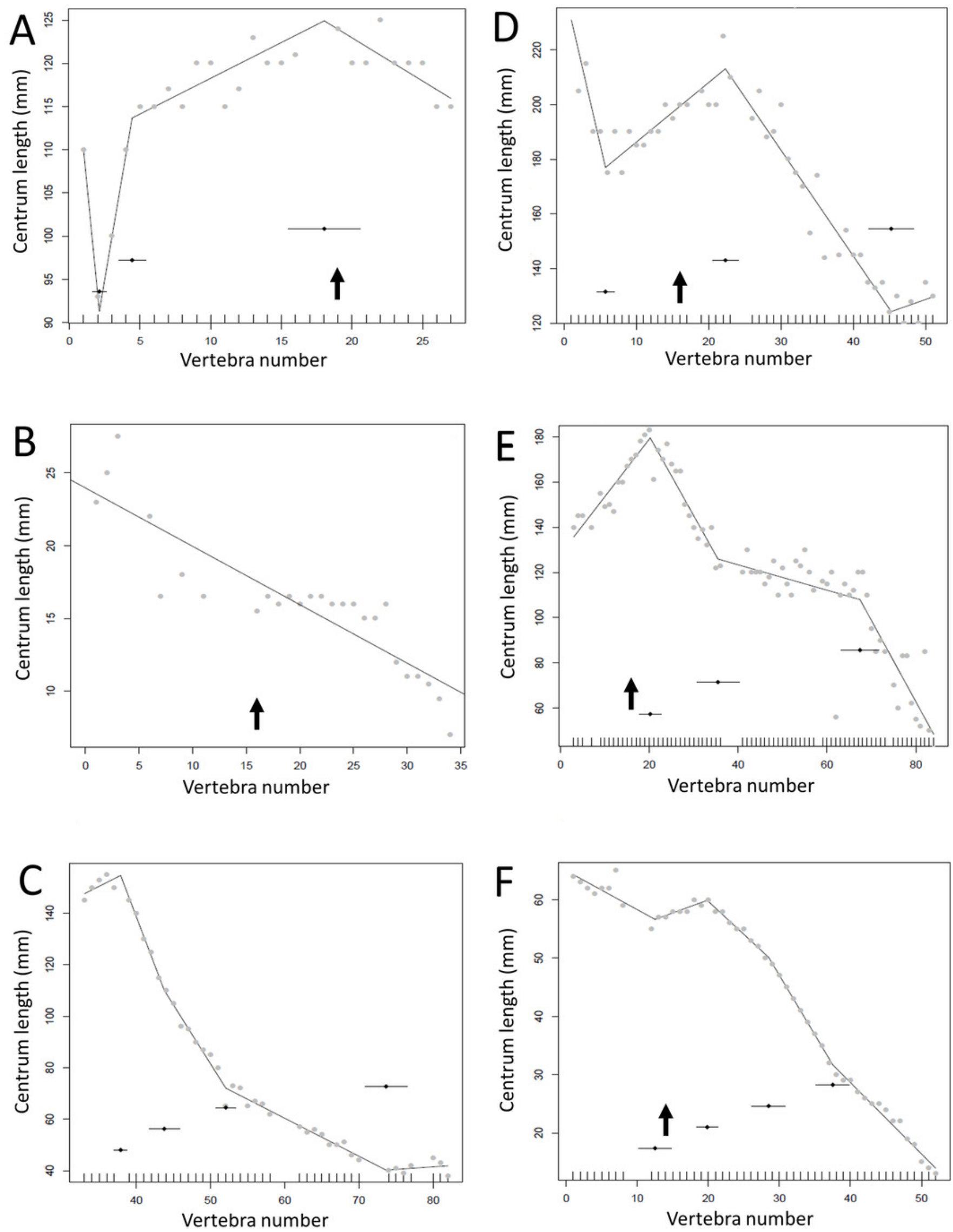


\section{Figure 7}

Figure 7. Regressions for centrum lengths within the tails for non-maniraptoran members of the Theropoda, A), Dilophosaurus, B) Ceratosaurus, C) Majungasaurus, D) Juravenator, E) Gorgosaurus, F) Tyrannosaurus.

Note that patterns of the two tyrannosaurs, Gorgosaurus and Tyrannosaurus, are most similar to each other and that the pattern of Juravenator is the most dissimilar from all other theropods. The predicted break points and their error bars are indicated at the bottom of each graph where these have been calculated, and the transition point (where known) is indicated by an arrow. 

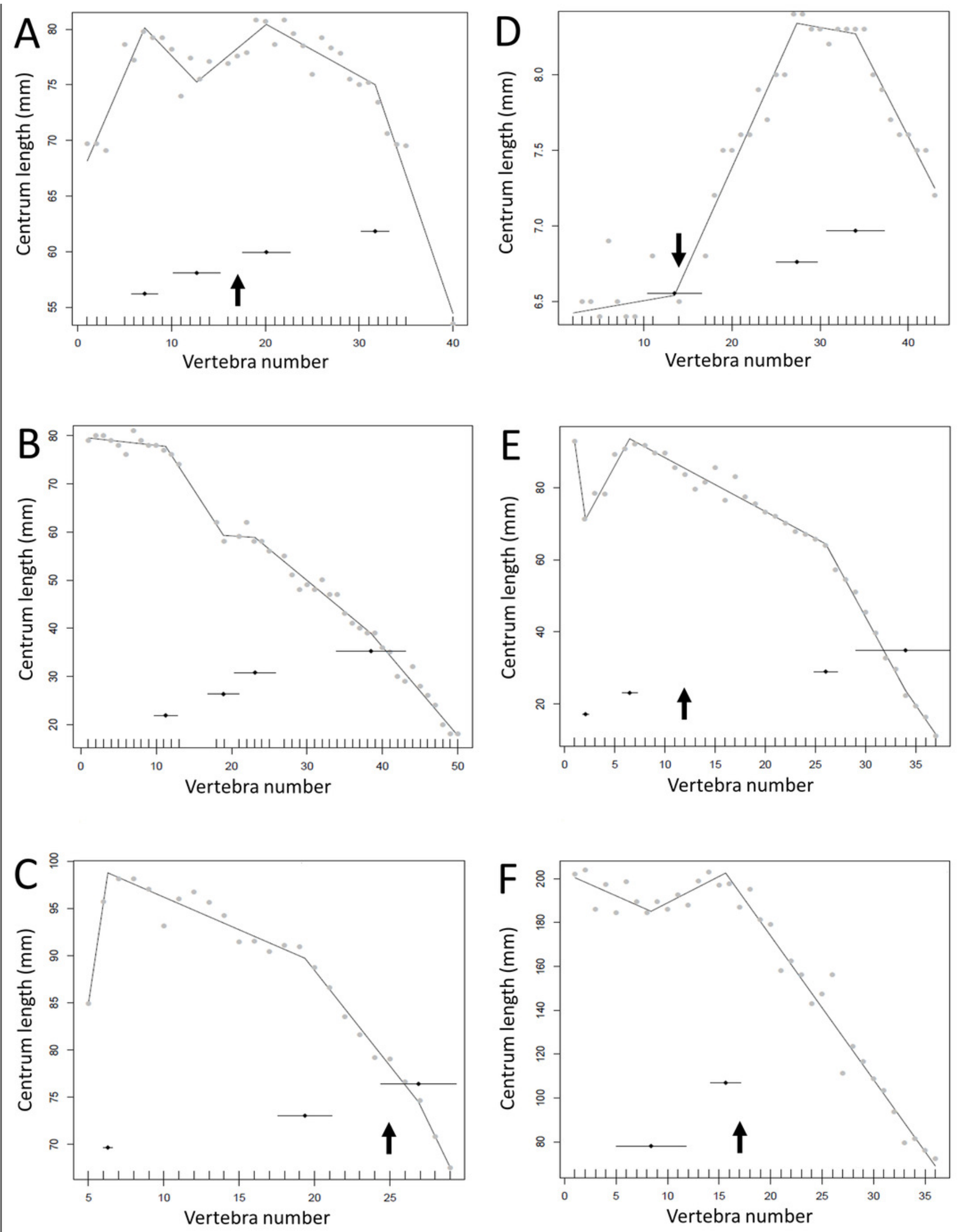


\section{Figure 8}

Figure 8. Regressions for centrum lengths within the tails for selected members of nonparavian Maniraptora A) Ornithomimus B) Ingenia, C) Nomingia.

The two oviraptorosaurs $(B, C)$ show strongly tapering tails. The predicted break points and their error bars are indicated at the bottom of each graph where these have been calculated, and the transition point (where known) is indicated by an arrow. 

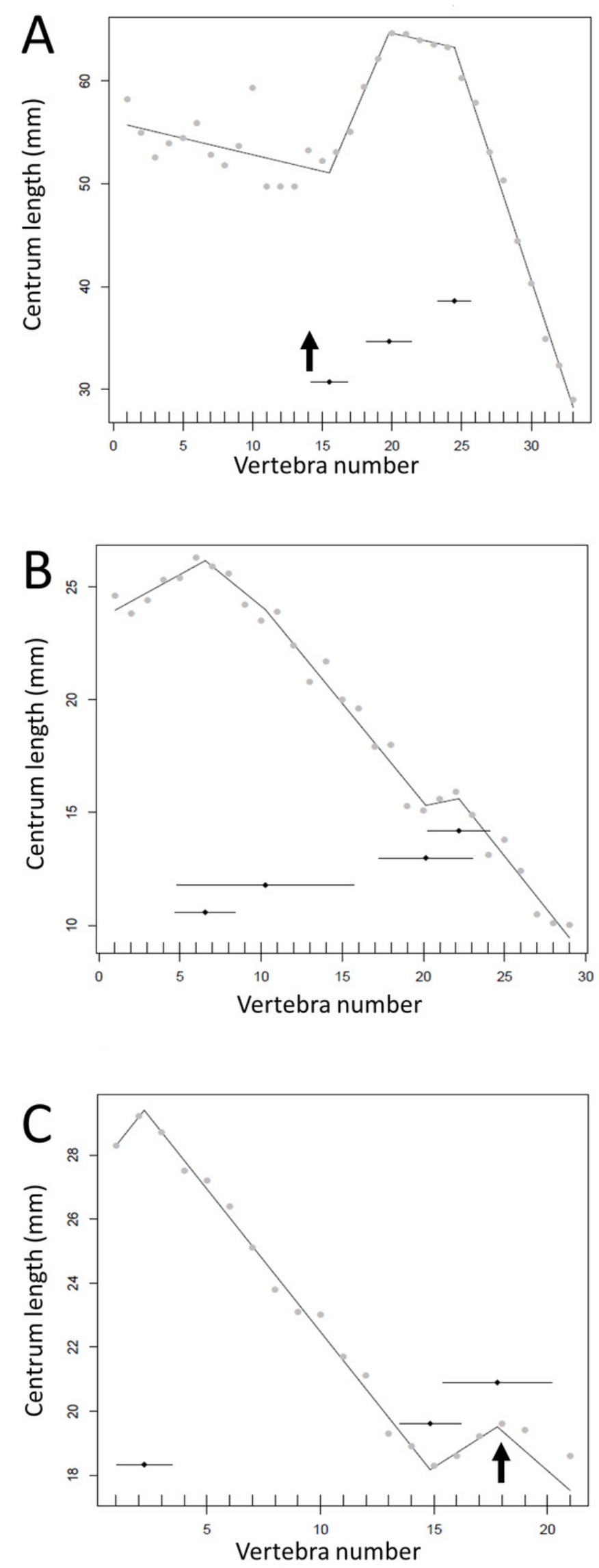

Peer] reviewing PDF | (2020:04:48148:3:0:NEW 3 Dec 2020) 
Figure 9

Figure 9. Regressions for centrum lengths within the tails for members of the Dromaeosauridae, A) Velociraptor IGM 100/25, B) Velociraptor AMNH 100-986, C) Velociraptor MPC 100/985), D) Deinonychus, E) Microraptor.

The predicted break points and their error bars are indicated the bottom of each graph

where these have been calculated, and the transition point (where known) is indicated by an arrow. 

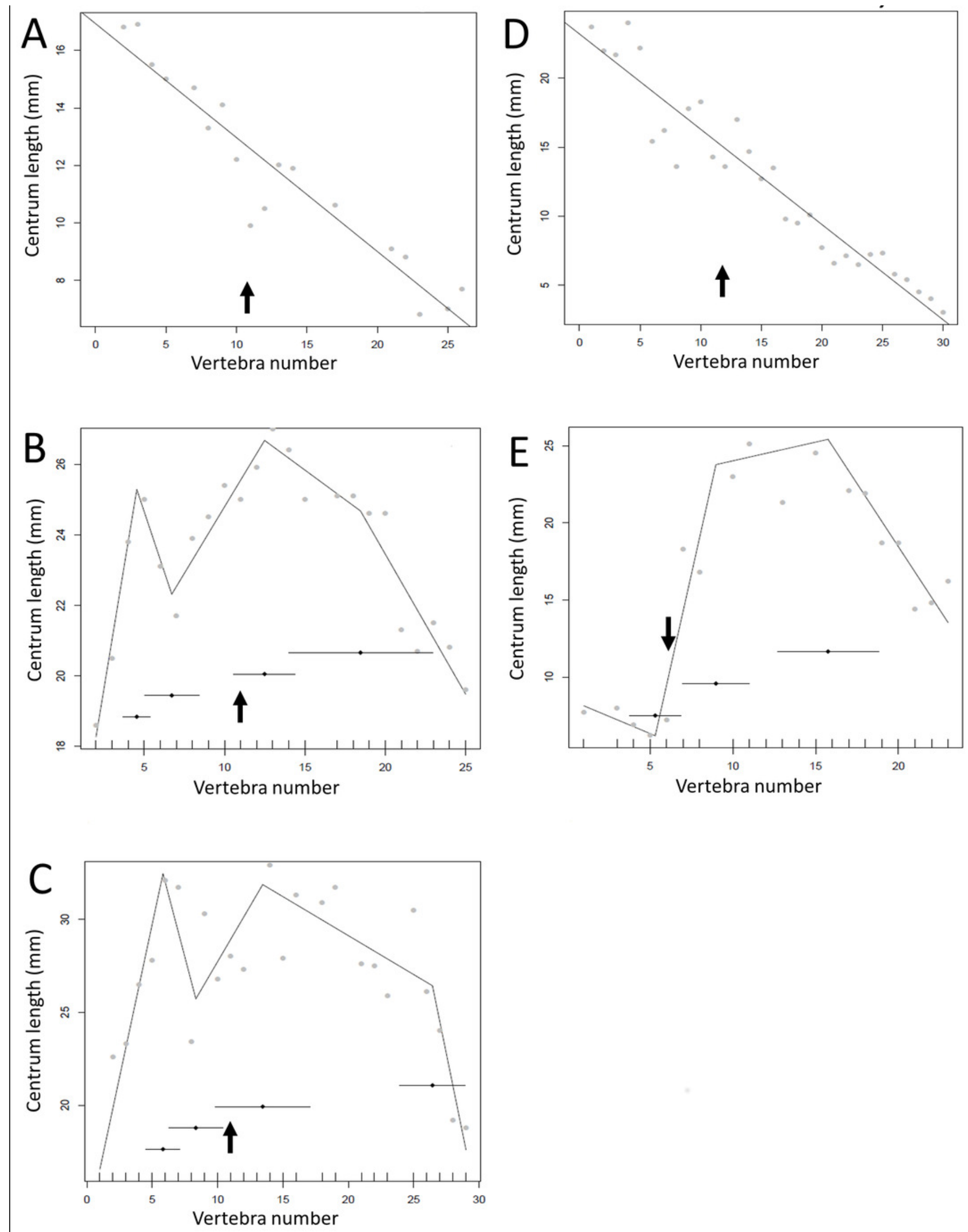


\section{Figure 10}

Figure 10. Regressions for centrum lengths within the tails for specimens of Archaeopteryx (A) BSPG1999150, B) WDC CGS100 C) $11^{\text {th }}$ Specimen) and Coelophysis (D) AMNH 7223, E) AMNH 7224, F) AMNH7229).

Archaeopteryx is a rare genus for which numerous specimens with complete tails are known. Although the general pattern observed across the specimens is similar, the pattern of each specimen is distinct. Despite all three Coelophysis specimens coming from the same locality, their tails differ markedly from each other. The predicted break points and their error bars are indicated at the bottom of each graph where these have been calculated, and the transition point (where known) is indicated by an arrow. 

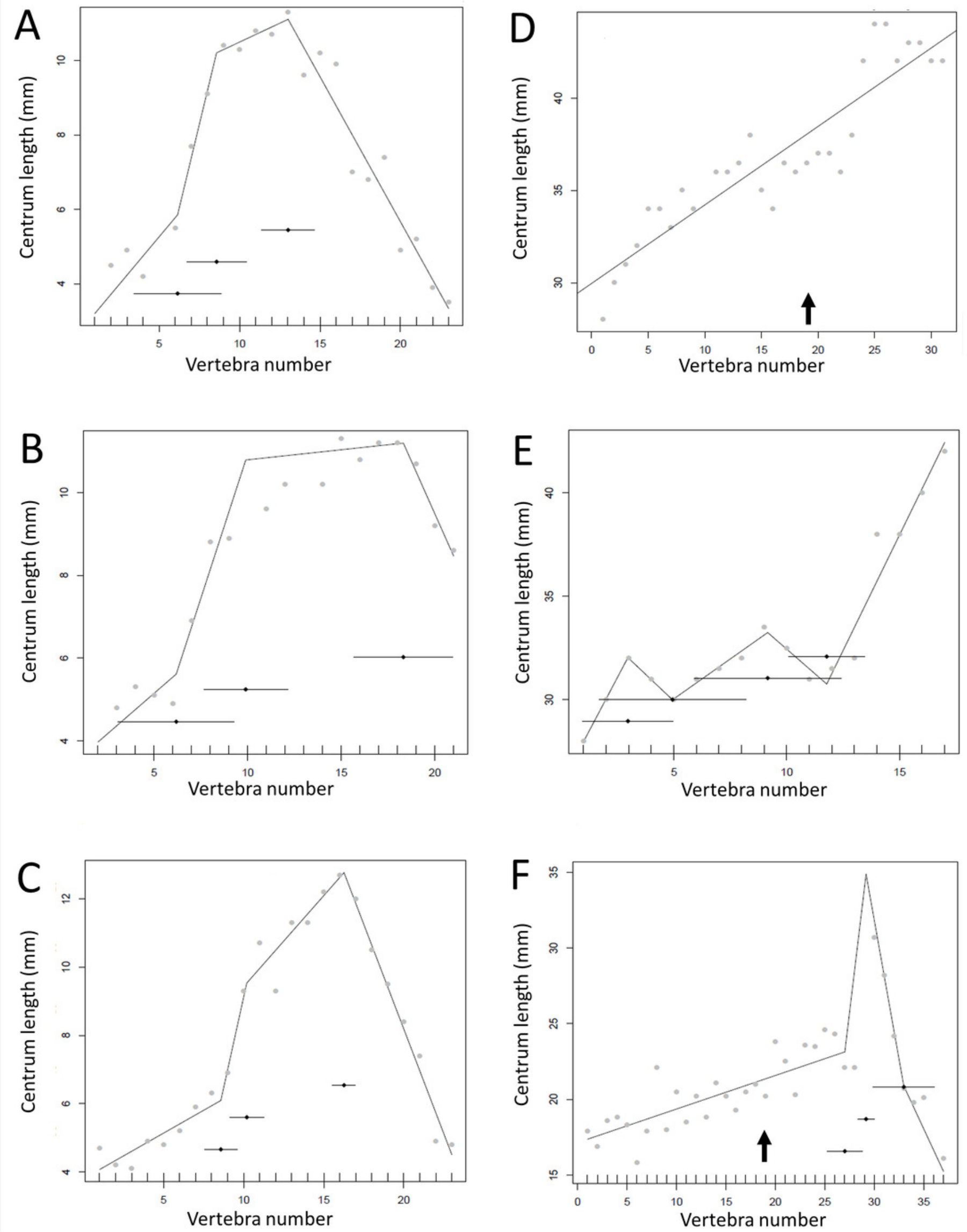


\section{Figure 11}

Figure 11. Regressions for centrum lengths within the tails for members of the Thyreophora, A) Kentrosaurus, B) Dyoplosaurus.

More than any other taxa in the study, the club bearing tail Dyoplosaurus would be expected to show a specialized pattern, and it does, although surprisingly the analysis did not recover break points in the series. The predicted break points and their error bars are indicated at the bottom of each graph where these have been calculated, and the transition point (where known) is indicated by an arrow. 

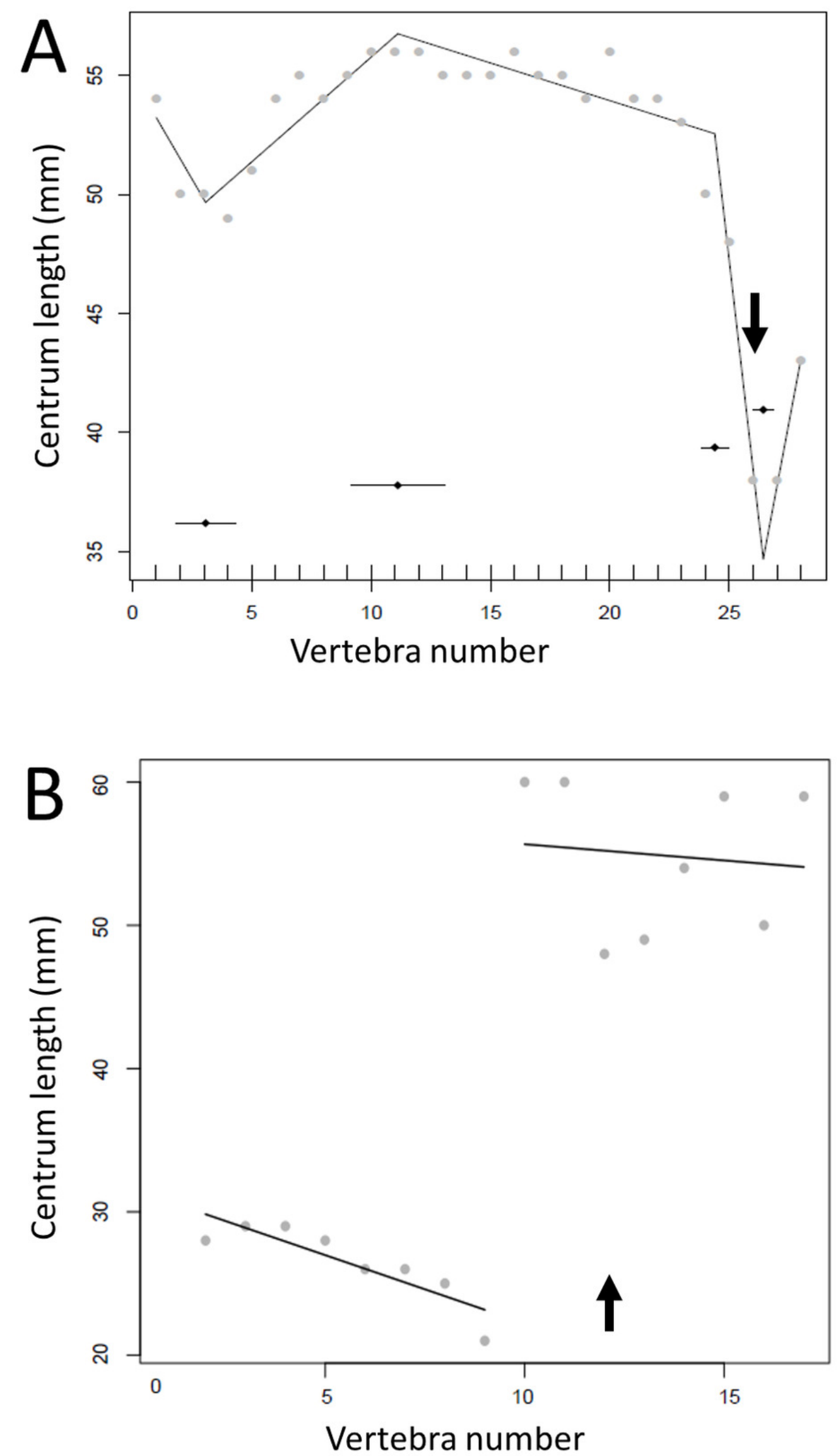


\section{Figure 12}

Figure 12. Regressions for centrum lengths within the tails for members selected nonhadrosauroid iguanodontians, A) Tenontosaurus, B) Ouranosaurus, C) Iguanodon) and hadrosaurs, D) Tethyshadros, E) Lambeosaurus).

Despite their overall similarity in form and ecology there are considerable differences between the patterns seen between these taxa. The predicted break points and their error bars are indicated at the bottom of each graph where these have been calculated, and the transition point (where known) is indicated by an arrow. 

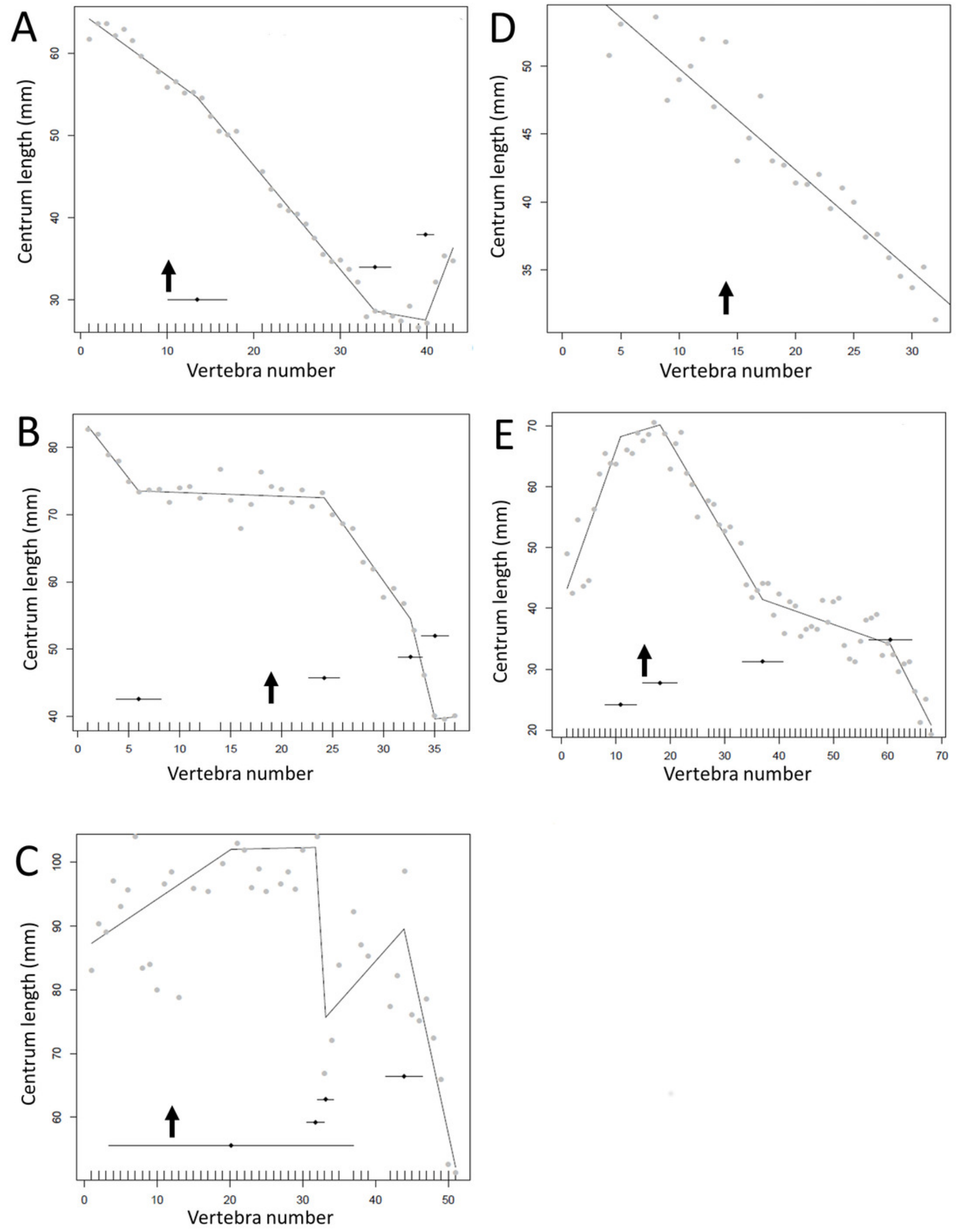


\section{Figure 13}

Figure 13. Regressions for centrum lengths within the tails for members of the Ceratopsia. A) Psittacosaurus AMNH 6253, B) Psittacosaurus AMNH 6254, C) Psittacosaurus GiSPS 100/606, D) Leptoceratops CMN 8887, E) [i]Leptoceratops [i

Relative to that of most other dinosaur groups considered, the ceratopsians pattern is closer to simple progressive tapering. The predicted break points and their error bars are indicated at the bottom of each graph where these have been calculated, and the transition point (where known) is indicated by an arrow. 

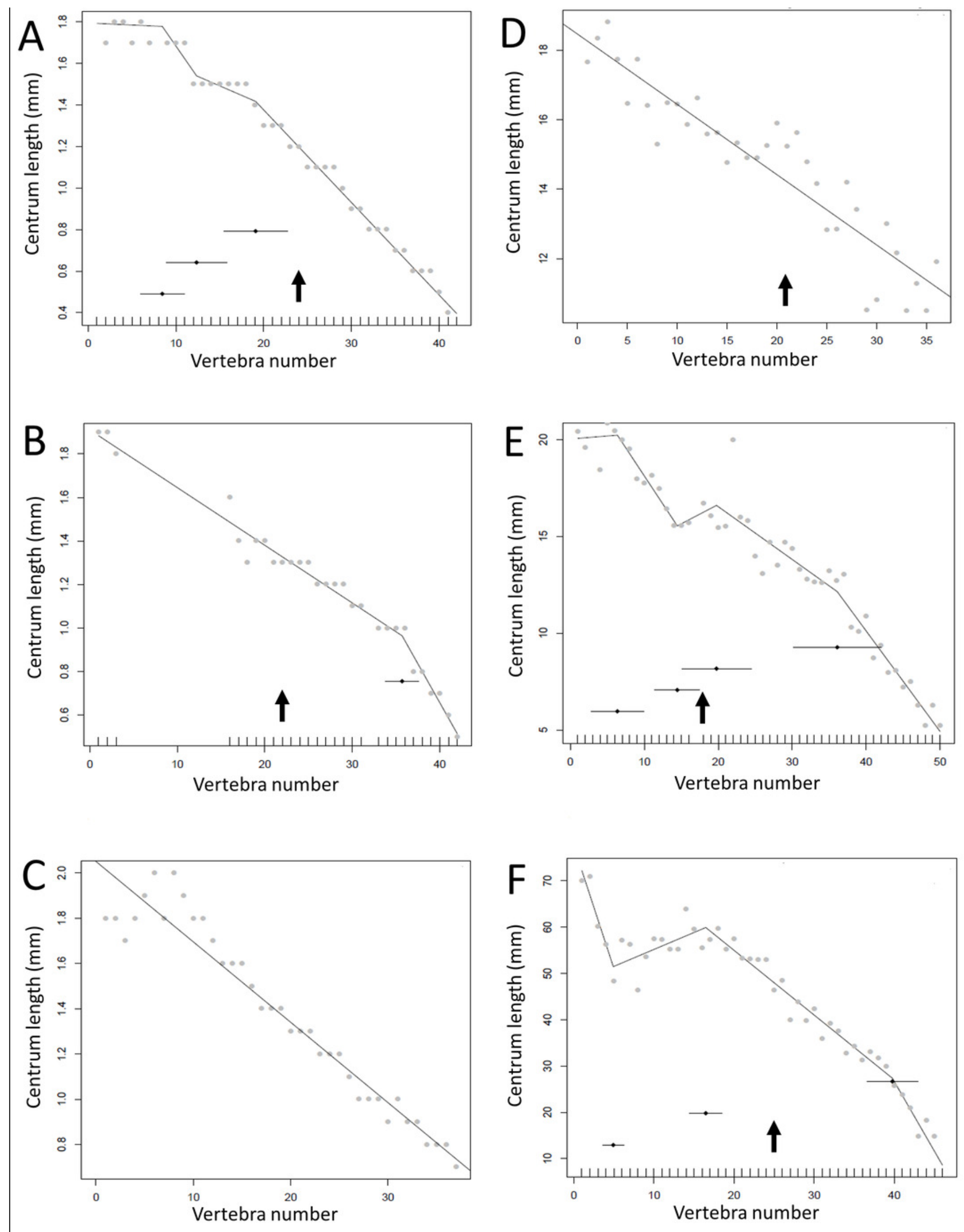